\title{
UNITARITY, DUALITY AND ABSORPTION; A GENERAL DISCUSSION AND A DEFINITE MODEL *
}

\author{
G. COHEN-TANNOUDJI and R. LACAZE, \\ Service de Physique Theorique, CEN-Saclay, Gif-sur-Ylette, France \\ F.S. HENYEY, D. RICHARDS** and W.J. ZAKRZEWSKI \\ Physics Dept., University of Michigan, Ann Arbor. Michigan, USA \\ G.L. KANE**** \\ Rutherford High Energy Laboratory, Chilton, Didcot, Berkshire, England
}

Received 24 February 1972

\begin{abstract}
We study the problem of incorporating unitarity effects into dual scattering amplitudes. Possible forms and interpretations for amplitudes which have dual properties consistent with experimental data (including the fixed-t zero structure, the dominance of peripheral resonances, etc,) are discussed. A detailed physical interpretation, in terms of absorption effects, is provided at all energies. $A$ specilic model is presented which demon strates that amplitudes of the desired kind do exist and that one can work with them.

The model is studied in detail for spinless particles (e.g. $\pi \pi$ scattering). Apart from at few technical difficulties the resulting amplitude can be studied at all energies and angles and behaves in all aspects essentially as we expect realistic scattering amplitudes (and data) to behave.
\end{abstract}

\section{INTRODUCTION}

Since the introduction of dual models for the scattering amplitude, the role of unitarity in determining the amplitude has been de-emphasized. Some qualitative features of unitarity are incorporated into duality, while others are usually ignored. It is the purpose of this paper to study some of the consequences of incorporating more aspects of unitarity into dual amplitudes.

One feature of unitarity is probably present in all dual models. The central concept of duality is a connection between the amplitude at low energy and the amplitude at high energy. It would be possible, in the absence of unitarity constraints,

* Research supported in part by the US Atomic Energy Commission.

** Physics Dept., Johns Ilopkins University, Baltimore, Maryland, USA.

*** J.S. Guggenheim Memorial Foundation Fellow, on Sabbatical leave from the University of Michigan. 
to choose the high-energy and low-energy behavior of the amplitude essentially independently. The only trouble would be terms proportional to $1 / s, 1 / s^{2}$, etc., as $s$ goes to infinity, in addition to the dominant (Regge) terms. However, these terms represent fixed poles in the angular momentum plane (at values of $j$ other than nonsense wrong signature). The fixed poles are excluded by unitarity (except possibly under highly unusual circumstances), and hence the low- and high-energy behaviors of the amplitude are related.

There are many other aspects of unitarity. One of the most obvious, is the width of resollances. Narrow resonance models, by their very nature, badly violate this aspect of unitarity. There are different approaches taken to remedy this defect. The most ambitious is the unitarization of the Veneziano model by use of loop diagrams of immense complication. Pending the outcome of this program, a more modest approach is likely to be useful. This approach is to write amplitudes which approximately satisfy selected constraints of unitarity. and may have parameters which are fitted phenomenologically. Such an approach can lead to scattering amplitudes which are qualitatively valid in all regions of all variables simultaneously. These amplitudes may contain features of the fully unitarized dual models which are not contained in the original Veneziano model.

There have been several attempts to include wide resonances in a dual model. We proposed one, which we call $s-x$ duality, in an earlier paper [1]. The most serious defect of $s-x$ duality is that it is difficult to evaluate numerically. In the present paper we give up some of the nice exact features of $s-x$ duality and replace them by the same features approximately holding in an easily computable amplitude. (Sufficient progress has been made since we began this work in computing the $s-x$ dual amplitude so that soon one can do away with this approximation.)

There is one more important aspect of unitarity which must be included in a model for any amplitude which has a hope of being phenomenologically successful. This teature is the absorption of the interior partial waves at high energy, and the related feature of inclasticity of high-mass resonances. There is still a great deal of controversy on the proper method of applying this correction. It is generally accepted, however, that these corrections are needed, and that they are represented by cuts in the angular momentum plane. In this paper we will include absorptive Regge cuts in our amplitude. We will not use any definite absorption prescription to gencrate these cuts, nor will we insist that their branch points have precisely the Amati-Fubini-Stanghellini value as is usual in absorption approaches; rather we will attempt to approximately reproduce those aspects of the cuts which have been found phenomenologically to be important.

One consequence of approximately unitarizing dual models, both in $s-x$ duality and in the model of this paper, is the high degree of arbitrariness in such models. This fact is in striking opposition to the Veneziano model, which has little freedom of variation*. It now appears that the uniqueness of the Veneziano model is a conse-

\footnotetext{
* Of course the Veneziano model is non-unique on the daughter level because of the possibility
} of adding satellite terms. 
quence of the unphysical assumption of narrow resonances. When this assumption is replaced by the physical assumption that the total width of a resonance be at least as large as its partial width for decay into some channel, we find that duality, crossing Regge behavior and analyticity are not sufficient to determine the amplitude. An exact inclusion of unitarity (which is still beyond our abilities) might fill in the missing information. Instead, however, we will restrict our model by phenomenological considerations; the freedom of the arbitrariness allows the model to qualitatively reproduce many expected features of data at all energies and angles.

When the reader sees the method of construction of the amplitude in this paper, he can easily construct very many other similar models. We have examined a handful of models slightly different from the one we present, and have found them inferior phenomenologically, even though they are equally good theoretically. Thus we will present our function without apologizing for several ad hoc assumptions we make. Any improvement, which is certainly possible, in the form chosen would be indeed welcomed.

\section{PHYSICS OF THE MODEL}

There are at least two reasons that the Veneziano model, unitarized, cannot be a good approximation to the physical scattering amplitude. Everybody is aware that the Veneziano resonance poles do not lie on the second sheet where they belong; it is not necessary to argue this point. Less well accepted, but equally important, is that the Veneziano model has only Regge-pole asymptotic behavior, rather than Regge pole plus cut (or cuts).

Let us briefly review the evidence for the necessity of Regge cuts, or more precisely, Regge cuts of the enhanced absorptive type*. The enhancement of absorption has been theoretically derived from a variety of viewpoints, and is not purely a phenomenological construct. Consistent theoretical pictures presently existing require that absorption either be universally absent, or be universally present. The latter viewpoint stresses the composite nature of the scattering particles. The presence of absorption (though not its detailed properties) is then independent of everything else, in particular of the nature of the exchange, or of the particular combination of hadrons which are scattering. Thus the existence of absorption in several situations provides a strong argument for its existence everywhere. The argument for the enhancement of absorption is similar. If absorption is enhanced over that given by elastic scattering alone in some situations, it is likely to be enhanced elsewhere.

There are two well-known situations in which enhanced absorption is clearly

\footnotetext{
* We introduce here the terminology enhanced absorption to refer to absorption with a strength larger than that due to elastic scattering alone, independently of the mechanism chosen by Regge poles at nonsense and/or wrong signature integers. Strong absorption has come to mean one particular type of enhanced absorption, that of the Michigan model (SCRAM) [2].
} 
suggested very strongly by the experimental data. The first of these is in reactions where $\pi$-exchange plays an important role, most notably in charged pion photoproduction. In these reactions there is a sharp forward peak of width $\Delta t \approx m_{\pi}^{2}$. This can be explained only by conspiring Regge poles (or fixed poles) or by Regge cuts. The pole conspiracy theory for these reactions has been discredited. With the Regge cuts the forward amplitude is directly proportional to the size of the cut. It is thus a rather simple matter to discover that the magnitude of the cut is significantly larger than that given by elastic absorption, and by studying the interference of the cut (assumed relatively structureless on a scale $\Delta t \approx m_{\pi}^{2}$ ) with pion exchange to find that the cut has the phase (approximately, at least) given by absorption [3].Enhancec absorption thus provides a very natural explanation.

The other phenomenon in which enhanced cuts are easily seen to be essential at high energy is that of the crossovers. The difference of the $\pi^{-} p$ and $\pi^{+} p$ elastic cross sections, that of $\mathrm{K}^{-} \mathrm{p}$ and $\mathrm{K}^{+} \mathrm{p}$, etc., vanish at $t \approx 0.15 \mathrm{GeV}^{2}$. These crossovers are due to an interference between the dominant Pomeranchuk amplitude, and an odd signature amplitude. This crossover must come from the vanishing of that part of the odd signature amplitude in phase with the Pomeranchon. Conventional pure Regge-pole models for the odd signature amplitude (such as the Veneziano model) put this crossover at $t \approx 0.6 \mathrm{GeV}^{2}$. Weak absorption places it at $-t \geqq 0.3 \mathrm{GeV}^{2}$, and only enhanced absorption can place it at sufficiently small $t$-values. A pure Regge pole model with a vanishing residue at the crossover point is well known to have difficulties with factorization [4], say; in a dual framework the situation is even worse since the whole structure of the amplitude is likely to be spoiled by the introduction of a zero independent of the signature ones.

Other evidence for enhanced absorption at high energy depends on more involved arguments (in particular the failure of factorization); or on detailed models of enhanced absorption such as the strong cut model; or is also consistent with other kinds of models. Universal enhanced absorption thus depends on considerations of theoretical consistency, as well as on phenomenological grounds.

It is perhaps worth pointing out that even though there is no compelling derivation of an absorption model, there is no theoretical or phenomenological evidence against the absorption effect; for example the frequent claim that the $\pi \mathrm{N}$ polarization data (elastic or charge exchange) cannot be described correctly in this approach, can easily be shown to be incorrect if proper attention is paid to phases [5], or to secondary cut contributions [6].

Absorption is the suppression of low partial waves. At low energies the same phenomenon occurs. In this case, the cause is somewhat different, although related. At high energies absorption is due, via unitarity, to competition with many other channels. At very low energies as we shall see, the suppression is due to unitarity bounds. At all energies, this phenomenon is related to the removal of flux from the incident beam by scattering. The difference is only in the relative amount of that scattering into the channel being studied.

At low energies the contributions from the lowest partial waves must be smaller 
that those for higher partial waves simply because the $2 l+1$ factor in the partial wave amplitude multiplies something bounded independently of $l$. Thus, for example when the $l=1$ partial wave in $\pi \pi$ scattering is resonating at $s=m_{\rho}^{2}$, the $l=0$ partial wave is at least a factor of three smaller.

It is a simple calculation to see that the P-wave contribution has a zero at $t=-\frac{1}{2}\left(m_{\rho}^{2}-4 m_{\pi}^{2}\right)=-0.26 \mathrm{GeV}^{2}$. In the process $\pi^{+} \pi^{-} \rightarrow \pi^{+} \pi^{-}$, even with a $90^{\circ}$ $\mathrm{S}$-wave phase shift the zero is at $t=-0.34 \mathrm{GeV}^{2}$, ref. [7]. These positions are to be compared to the zero (in the residue of the pole at $s=m_{\rho}^{2}$ ) at $t \approx-0.6 \mathrm{GeV}^{2}$ in the Veneziano model. Another example in $\pi \pi$ scattering of a suppression of low partial waves is the absence of a clearly observable $\rho^{\prime}$ meson. In a model such as the Vene ziano model, without suppression effects, the $\rho$ ' should be clearly visible in the isospin one $\pi \pi$ channel. Experimentally this is not the case, indicating a suppression.

The same effect occurs in $\pi \mathrm{N}$ scattering. The helicity non-flip amplitude at low energies has a zero at small $t$-values. One can easily find that at $s=m_{\Delta}^{2}$ the zero of the non-flip amplitude is near $t=-0.07 \mathrm{GeV}^{2}$. All of the other prominant $\pi \mathrm{N}$ resonances, i.e. those used by Dolen, Horn and Schmid [8] in their successful analysis of finite energy sum rules, also have their zeros at small $t$-values, $t \approx-0.2 \mathrm{GeV}^{2}$.

We have seen that both at high energy and at low energy, there is a suppression of low partial waves. In both cases the zero in $t$ of the amplitude is at roughly the same place. The connection of the $t$-dependence of an amplitude at high and low energies is the principle feature of duality. Thus the two aspects of suppression are related, not only by both being consequences of unitarity, but also by the duality relationship.

These considerations about hte role of absorption at low energies indicate that we can give a simple physical interpretation at all energies to a model with poles plus absorption. As we will see in more detail when we discuss our results, the absorption interpretation at low energies is very attractive.

For example, at low and intermediate energies the absorption can be thought of as arising from the opening of inelastic channels. Thinking of these as two-body channels for simplicity, we expect that they first contribute in the lowest partial waves, from centrifugal barrier arguments. Thus they lead to a suppression of the central resonances (low spin and higher mass) relative to the peripheral ones in agreement with observation. It is this reduction of the central resonances by absorption, at all energies, which leads to the observed duality of experimental data, with similar structure in $t$ at all energies.

To see this from another point of view, consider the position of the zeros [9] in momentum transfer of the imaginary parts of the $s$-channel helicity amplitudes $M_{++}$ and $M_{+-}$(non-flip and flip respectively) for $\pi^{-} p \rightarrow \pi^{\circ} \mathrm{n}$. At high energies the crossover of $\pi^{ \pm}$p indicates that $\operatorname{Im} M_{++} \approx 0$ at $-t=0.2$, while the dip in $\mathrm{d} \sigma / \mathrm{d} t$ indicates the dominant amplitude $M_{+_{-}}$vanishes near $-t=0.6$. Both amplitudes are dominated by $\rho$-exchange. Thus, experimentally, amplitudes have a structure depending in an important way on both $t$-channel (the $\rho$-exchange) and $s$-channel (the helicity flip) quantum numbers. 
Each of these amplitudes has a similar structure at high and low energies [8], i.e. a dual structure. Thus any dual model that is consistent with the data must have amplitudes which depend on both $s$-and $t$-channel quantum numbers.

The absorption model can give the desired effect in a natural way at both high and low energies. At high energies the non-flip amplitude is absorbed more that the flip because the former allows a head-on collision while the latter does not $\left(M_{+}\right.$. vanishes in the forward direction from angular momentum conservation), so one finds [2] a zero at $-t \approx 0.2$ in $M_{++}$and at $-t \approx 0.6$ in $M_{+\ldots}$. At low energies the central resonances are absorbed relative to the peripheral ones by the opening of inelastic channels in the low partial waves, so the peripheral resonances can dominate and give the same structure in $t$ as at high energies.

At this point we can discuss the most important feature of our work. In the above analysis we have considered the entire amplitude at high energies Regge pole plus absorptive cut. At low energies we considered mainly resonances. We find that the resonances are dual to the pole plus cut, not to the pole alone. The Regge pole alone, if it has any zero, has it at a much larger $t$-value than either the resonances or the entire high-energy amplitude. It is untenable that the resonances and Regge poles are dual, while the Regge cut is a correction only, dual to something else, for example background of some sort.

One can see the difference between the actual role of cuts in duality as described above, and that envisioned by the model of one-loop duality diagrams. For these diagrams, in meson-meson scattering, the Regge cut is dual to the pomeron (or something with vacuum quantum numbers) in the crossed channel, not to part of the resonances. In the entire dual loop model this problem may be overcome. Individual two-loop diagrams contain Regge poles as well as Regge cuts [10].

Moreover, the simplest duality diagram with an absorptive cut (rather than a Regge-Regge cut), contains two loops (if the pomeron is dual to the one-loop Regge cut ).

Now we shall make a simplifying assumption which allows us to perform the nu. merical calculation. This assumption is that the amplitude may be decomposed into a sum of dual terms [11]. Each terms has one behavior (either pole or cut) in one channel, and one behavior in another channel. It may be exotic in the third channel. Examples of such terms, with our notation for them, are:

$$
\begin{array}{ll}
\left(P_{s} P_{t}\right) & \text { poles in both the } s \text {-and } t \text {-channels, } \\
\left(C_{s} C_{t}\right) & \text { cuts in both the } s \text {-and } t \text {-channels, } \\
\left(P_{s} C_{t}\right) & \text { pole in the } s \text {-channel and cut in the } t \text {-channel. }
\end{array}
$$

If the Veneziano expression $\Gamma\left(-\alpha_{s}\right) \Gamma\left(-\alpha_{t}\right) / \Gamma\left(-\alpha_{s}-\alpha_{t}\right)$ had finite width resonances, it might be an acceptable $\left(P_{s} P_{t}\right)$ term.

This assumption may appear to be a minor technicality. However, the immediate consequences are not minor, and may turn out to be incorrect. We have shown above that the $s$-channel resonances are dual to the sum of $t$-channel poles plus $t$-channel 
cuts. Therefore, the amplitude must contain the terms $\left(P_{s} P_{t}\right)$ and $\left(P_{s} C_{t}\right)$. The term $\left(P_{s} P_{t}\right)$ has either no zero, or the zero at too large a $-t$ value; $\left(P_{s} C_{t}\right)$ interferes destructively with $\left(P_{s} P_{t}\right)$ producing a zero in non-flip amplitudes at $t \approx-0.2 \mathrm{GeV}^{2}$. at both high and low energies. However, $\left(P_{s} P_{t}\right)-\left(P_{s} C_{l}\right)$ does not have correct crossing properties. $\left(P_{s} P_{t}\right)$ crosses into the same type term under $s \leftrightarrow t$ but, $\left(P_{s} C_{t}\right)$ crosses into a term of the term $\left(C_{s} P_{t}\right)$. Thus the amplitude must also contain $\left(C_{s} P_{t}\right)$ also interfering destructively with $\left(P_{s} P_{t}\right)$. Now, however, the expression $\left(P_{s} P_{t}\right)$ $-\left(P_{s} C_{t}\right)-\left(C_{s} P_{t}\right)$ no longer has a zero at $t \approx-0.2 \mathrm{GeV}^{2}$. This can be remedied only by including a term $+\left(C_{S} C_{t}\right)$, interfering destructively with $\left(C_{S} P_{t}\right)$. This last term crosses properly. Thus the entire non pomeron amplitude can be written as:

$$
F(s, t)=\left(P_{s} P_{t}\right)-\left(P_{s} C_{t}\right)-\left(C_{s} P_{t}\right)+\left(C_{s} C_{t}\right)
$$

This may be written as $\left((P-C)_{S}(P-C)_{t}\right)$. This form is very suggestive. It implies that the best calculation would be to start from an acceptable $\left(P_{S} P_{t}\right)$ term, and simultaneously calculate absorption corrections in both channcls. Such a program is, however, considerably more ambitious than the calculations described in this paper.

Our assumption has led to an amplitude which does not appear to be resonance dominated at low energies. The $P_{s}$ terms contain the resonances while the $C_{s}$ terms contain background. At high energies both $\left(P_{s} P_{t}\right)-\left(P_{s^{\prime}} C_{t}\right)$ and $\left(C_{s} P_{t}\right)-\left(C_{s} C_{t}\right)$. have roughly the same $t$-dependence, which in turn is roughly the $t$-dependence of the amplitude. Thus the resonances are dual in shape to the high-energv amplitude. but not in size. In a comparison, for example using finite energy sum rules, the size is much more sensitive to the details of the calculation than is the shape. The assumption of resonance dominance at low energies is popular, and has a moderate amount of experimental support. Only a careful phenomenological analysis can distinguish it from the decomposability assumption which we make here.

The most satisfactory $\left(P_{s} P_{t}\right)$ terms are of the $s-x$ dual type [12], and are difficult to compute. Therefore we must approximate this tem by something less satisfactory. We could, for example, use a Veneziano formula with ancestors, but such an approximation is contrary to the usual spirit of duality. We have therefore used a completely different sort of approximation which we now describe. We have a great deal of freedom in choosing our terms, so we can write $\left(P_{s} P_{t}\right)-\left(C_{s} P_{t}\right)^{\prime} \approx 0$ for large $s$ where $\left(C_{s} P_{t}\right)^{\prime}$ is a term of the $\left(C_{s} P_{t}\right)$ type, but chosen in form and size to have roughly the same $t$-channel Regge pole as $\left(P_{s} P_{t}\right)$. Similarly $\left(P_{s} C_{t}\right)^{\prime}-\left(C_{s} C_{t}\right)^{\prime} \approx 0$ for large $s$. The combination $\left(P_{s} P_{t}\right)-\left(C_{s} P_{t}\right)^{\prime}-\left(P_{s} C_{t}\right)^{\prime}+\left(C_{s} C_{t}\right)^{\prime}$ crosses properly, and is therefore small for large $t$ as well as large $s$. It is reasonable to assune that this expression can be made very small everywhere by the proper choice of the primed terms. Thus by subtracting this very small expression from the amplitude we arrive at an approximate amplitude without any term of $\left(P_{s} P_{t}\right)$ type.

It is in this form that we will calculate the amplitude:

$$
F(s, t)=\left(P_{s} C_{t}\right)+\left(C_{s} P_{t}\right)-\left(C_{s} C_{t}\right)
$$


where the various components have the same behavior as in eq. (1), but can be different in size and can be obtained by redefining the pole residues and the cut discontinuities.

This form can be interpreted in terms of duality concepts, although not in as compelling a way as the four term expression from which it was derived. The first term has resonances at low $s$, while the second has the $t$-channel Regge pole. According to the ideas of duality, the sum of the first two terms involves double counting [8].

As we show in the next section, on a definite model, the third term is present in order

(i) to remove this double counting objection at lower energies,

(ii) to provide cut corrections with the correct absorptive sign.

In the following sections we will propose formulas for the $P C, C P$, and $C C$ terms which phenomenologically contain the features we have described, examine the successes and shortcomings of this calculation, and suggest an improvement in the form of our expressions.

\section{CONSTRUCTION OF A DEFINITE MODEL.}

As discussed above, because of the computational difficulty in obtaining a good pole-pole dual amplitude we have constructed a simplified version of what we expect to be the best model. For this reason we do not expect, when applying our model, to reproduce a best fit to experimental data. Our intention is to produce an explicit model amplitude which can be in qualitative agreement with possible data at all energies and angles. We have chosen to apply our model to $\pi \pi$ scattering for several reasons [13]:

this reaction does not involve any spin complications;

some pieces of low-energy experimental information exist;

from factorization arguments, the high-energy Regge phenomenology is rather well constrained;

the reactions which are actually observed show very general features which we expect also to occur in $\pi \pi$ scattering. It is then interesting to study the $\pi \pi$ amplitudes as a laboratory before extension to processes with spin;

this reaction has been extensively studied on theoretical grounds, and many useful constraints have been derived from fïst principles;

various dual models have already been proposed with which we can compare our own model.

In addition we have not restricted our investigation to $\pi \pi$ scattering, but following Lovelace's [14] suggestion. we have extended them by pure analytic continuation to the study of $\bar{p}$ annihilation at rest into three pions.

In this section we shall first exhibit explicit forms for the various components of the amplitude and briefly discuss their mathermatical properties; then we shall explain how the parameters can be determined, and finally we shall display and discuss the results obtained. 


\subsection{Construction and mathematical properties of the components of the amplitude}

The various pion-pion scattering amplitudes can be written in terms of the $\pi^{+} \pi^{-}$ elastic scattering amplitude, $A(s, t)$. All the crossing symmetry and isospin invariance conditions are given in the following equations:

$$
\begin{array}{ll}
A(s, t)=A(t, s), & A^{I_{s}=0}(s, t)=\frac{3}{2}\left[A(s, t)+A(s, u)-\frac{1}{3} A(t, u)\right], \\
A^{I_{s}=1}(s, t)=A(s, t)-A(s, u), & A^{I_{s}=2}(s, t)=A(t, u),
\end{array}
$$

with $s+t+u=4 m_{\pi}^{2}$.

Following the Freund-Harari conjecture [15] and the general duality framework we separate in $A(s, t)$ the pomeron and non-pomeron contribution

$$
A(s, t)=P(s, t)+F(s, t) .
$$

(i) The non-pomeron contribution $F(s, t)$. In the preceeding section we have proposed a structure for this non-pomeron contribution (eq. (2)) which we rewrite explicitly exhibiting a scale parameter in front of the $\left(C_{s} C_{t}\right)$ term

$$
F(s, t)=\left(P_{s} C_{t}\right)+\left(P_{t} C_{s}\right)+\Lambda\left(C_{s} C_{t}\right) .
$$

It is a rather easy matter to build the above mentioned contributions: the Veneziano formula provides us with a kernel from which we can generate classes of such functions. The smoothing procedure of Martin [16] consists in taking the convolution of the Veneziano function with a given distribution. Applying this technique on one or both trajectories allows us to get $(P C)$ or $(C C)$ terms. To be more explicit we have chosen to use the following forms

$$
\begin{gathered}
\left(P_{s} C_{t}\right)=\int_{0}^{\infty} \mathrm{d} \lambda \int_{a}^{b} \mathrm{~d} \mu \mathrm{e}^{-\lambda} \sqrt{(b-\mu)(\mu-a)} \frac{\Gamma(1-\alpha(s)) \Gamma\left(-\left(\alpha_{0}-1\right)(1+\lambda / q)-\mu t\right)}{\Gamma\left(-\alpha(s)-\left(\alpha_{0}-1\right)(1+\lambda / q)-\mu t\right)} \\
\left(C_{s} C_{t}\right)=\int_{0}^{\infty} \mathrm{d} \lambda \int_{a}^{b} \mathrm{~d} \mu \mathrm{e}^{-\lambda} \sqrt{(b-\mu)(\mu-a)} \frac{\Gamma\left(-\left(\alpha_{0}-1\right)\left(1+\lambda / q^{\prime}\right)-\mu s\right) \Gamma\left(-\left(\alpha_{0}-1\right)\left(1+\lambda / q^{\prime}\right)-\mu t\right)}{\Gamma\left(-2\left(\alpha_{0}-1\right)\left(1+\lambda / q^{\prime}\right)-\mu s-\mu t-1\right)}
\end{gathered}
$$

where $\alpha(s)$ is a complex $p-\mathrm{P}^{\prime}$ exchange degenerate Regge trajectory, $\alpha_{\mathrm{o}}=\alpha(0)$, and $a, b, q$ and $q^{\prime}$ are real parameters characterizing the model. The $\lambda$ integrations converge at infinity since the integrands for large positive $\lambda$ are bounded by $\lambda^{N} \mathrm{e}^{-\lambda}$. With $\alpha_{\mathrm{o}} \leqslant 1$, and $q, q^{\prime}, a, b>0$ all the integrals converge at least for $s$ and $t \leqslant 0$, in which region eqs. ( 5 and 6 ) define real analytic functions. 
In $\left(P_{s} C_{t}\right)$ the analyticity properties in $s$ are controlled by those of $\alpha(s)$ : one has a cut starting from the elastic threshold as in the trajectory function, and one has poles whenever $\alpha(s)$ equals an integer $n$. Since the residues of these poles in the integrand are polynomials in $t$ of degree $n$, and since the $\lambda$ - and $\mu$-integrals converge, the residue of the pole $\alpha(s)=n$ in $\left(P_{s} C_{t}\right)$ is also a polynomial in $t$ of degree $n$, which insures that at a finite mass one has a finite number of resonances. With $\operatorname{Im} \alpha(s)>0$ one insures that the resonance poles are actually second sheet poles*.

In $\left(P_{s} C_{t}\right)$ (and the same applies to $\left.\left(C_{s} C_{t}\right)\right)$ the singularities in $t$ come from the poles of the $\Gamma$-function depending on $t$ in the numerator of the integrand.Through the integrations these poles are smeared into cuts. Applying end point singularity reasoning one finds the positions of the $t$-branch points:

$$
t_{n}=\left[n-\left(\alpha_{0}-1\right)\right] / b, \quad\left[n-\left(\alpha_{0}-1\right)\right] / a, \quad n=0,1,2, \ldots
$$

Making $a=0$ sends half of these branch points to infinity; the branch points $\left[n-\left(\alpha_{0}-1\right)\right] / b$ can then be interpreted as equally spaced inelastic thresholds in the $t$-channel. The $\mu$-integration transforms the poles in the $\Gamma$-function in to square root branch points and the $\lambda$-integration provides a behaviour near $t_{n}$ of the type $\left(t-t_{n}\right)^{\frac{3}{2}}$

In order to compute the functions on the cuts in $t$ one can use dispersion relation techniques (with principal part integrations to get the real part); it is easier to use a complex contour $\mu$-integration (for instance a semi-circle running from $a$ to $b$ in the complex $\mu$-plane). This technique allows one to define the functions in terms of integrals which do converge for all values of $s$ and $t$ except at the thresholds where the behaviour is known anyway.

The asymptotic behavior of $\left(P_{s} C_{t}\right)$ and $\left(C_{s} C_{t}\right)$ where $s$ or $t$ go to infinity can be studied rather easily; we give here qualitative arguments which are justified a posteriori by explicit study in the appendix and by actual numerical computations. When $s$ goes to infinity the integrand in $\left(P_{s} C_{t}\right)$ is Regge behaved since it is a Veneziano amplitude ${ }^{* *}$; since one integrates over the intercepts and the slope of the Regge-pole trajectory one gets a behavior typical of a logarithmic Regge-cut contribution:

$$
\left(P_{s} C_{t}\right) \underset{s \rightarrow \infty}{\rightarrow} \frac{(-s)^{\alpha_{c}(t)}}{A+B \log (-s)}
$$

with $\alpha_{\mathrm{c}}(0)=\alpha_{0}$ (the $\lambda$-integration starts from 0$)$ and $\alpha_{\mathrm{c}}^{\prime}(0) \approx \frac{1}{2}(b+a)\left(\frac{1}{2}(b+a)\right.$ is the value of $\mu$ for which the weight function of the $\mu$-integration takes its maximum value). The same type of asymptotic behaviour in $s$ occurs for $\left(C_{s} C_{t}\right)$.

When $t$ goes to infinity it can be shown that $\left(P_{s} C_{t}\right)$ is Regge behaved,

* With the parameters which we have chosen we have numerically checked that the residues of the resonance poles on the parent and the first nine daughter trajectories are positive.

** Provided that $\alpha(s)$ is asymptotically linear with an imaginary part going to infinity slower than the real part. 


$$
\left(P_{s} C_{t}\right) \underset{|t| \rightarrow \infty}{\longrightarrow}(-t)^{\alpha(s)} \Gamma(1-\alpha(s)) \beta(s)
$$

where $\beta(s)$ is defined as a convergent integral over $\lambda$ and $\mu$.

Finally, we note that the usual procedure for writing Veneziano terms is to be followed here; whenever an amplitude would contain a standard Veneziano term we simply replace it by $(P C)+(C P)+\Lambda(C C)$. Thus our amplitude satisfies exact exchange degeneracy, in particular any amplitude which would be purely real in a Veneziano model will be real here. We will also have the usual linear signature zero in the Regge pole at the point where $\alpha_{\rho}=0$. But because of the absorption effects this zero (near $-t \approx 0.5$ ) will not have any detectable effects on the final amplitudes, as will always be the case for helicity non-flip amplitudes.

A property of our functions is related to the fixed angle behavior at high energy. One knows that the Veneziano amplitude does not explode when $s$ and $t$ go simultaneously to infinity $-t / s \leqslant 1$. Since in $\left(P_{s} C_{t}\right)$ we integrate over arguments of the $\Gamma$-function depending on $t$ which go to infinity the resulting function, even in the physical region $(|t|<s)$, is spoiled by the spurious exploding contributions of the Veneziano integrand. This difficulty is a direct consequence of the bad features of our kernel. We postpone to the next section the discussion of possible improvements of our model to get rid of this difficulty; in view of all the other desirable properties satisfied by our model amplitude, we have not tried to technically solve this problem for the present calculations. As a consequence we have mainly considered forward amplitudes at high energies. This trouble also gives rise to bad behavior of the low partial waves as the energy increases.

(ii) The pomeron contribution. We have not tried at all to construct a dynamical model for the pomeron contribution, but we have introduced this contribution in order to enlarge our domain of investigations to elastic scattering. The physical requirements which have guided us when building this contribution are mainly crossing and duality. From the Freund-Harari [15] point of view, the pomeron is completely decoupled from the resonance-Regge duality. Such a pomeron in a s-t crossing symmetric reaction can then be written as

$$
P(s, t)=H(s, t)+H(t, s)
$$

that is that the contribution of the pomeron in $\pi^{+} \pi^{-}$elastic scattering can be split into the exchanged pomeron $H(s, t)$ and the direct channel pomeron $H(t, s)$; $H(s, t)=H(u, t)$ in order that the pomeron has a positive signature. We then propose a form for the exchanged pomeron corresponding to a Regge-pole high-energy behavio

$$
H(s, t)=-\frac{\sigma_{\infty}}{\pi}(s-u) f(t) \frac{\left.\left\lfloor\left(\left(4 m_{\pi}^{2} \cdots s\right)^{\frac{1}{2}}+A\right)^{2 \alpha_{p}(t)-2}-\left(\left(4 m_{\pi}^{2}-u\right)^{\frac{1}{2}}+A\right)\right)^{2 \alpha_{\mathrm{p}}(t)-2}\right]}{2\left(\alpha_{\mathrm{p}}(t)-1\right)}
$$


where $\alpha_{\mathrm{p}}(t)$ is a Pomeranchuk trajectory, $\alpha_{\mathrm{p}}(0)=1, A$ a positive parameter which controls the way in which the pomeron contribution reaches its asymptotic regime, and $f(t)$ a residue function. Giving to $\alpha_{\mathrm{p}}(t)$ and $f(t)$ the standard analyticity properties of trajectory and residue functions guarantees that the pomeron contributions have the required analyticity properties. It is again the duality requirement which provides us with a hint for the parametrizations of $\alpha_{\mathrm{p}}(t)$ and $f(t)$ for positive $t$. Since we want the pomeron to be decoupled from duality we want the direct channe pomeron to contribute a non-resonating background falling very fast at high energy in order for it not to contribute in finite energy sum rules. Actually, by choosing a bounded trajectory for $\alpha_{\mathrm{p}}(t)$, for instance

$$
\alpha_{\mathrm{p}}(t)=1-\left(\left(4 m_{\pi}^{2} t\right)^{\frac{1}{2}}-2 m_{\pi}\right) /\left(\left(4 m_{\pi}^{2} \cdots t\right)^{\frac{1}{2}}+B\right)
$$

and for $f(t)$ a function falling to zero faster than any inverse power of $t$, such as

$$
f(t)=\exp \left(-\left(\left(z_{1}-t\right) / z_{2}\right)^{\frac{1}{4}}-\left(z_{1} / z_{2}\right)^{\frac{1}{4}}\right)
$$

we make sure that the direct channel pomeron contribution is not only superconvergent but in fact decreases faster than any inverse power of $s$ (we call it a hyperconvergent pomeron).

\subsection{Determination of the parameters}

In the appendix we have summarized all the mathematical properties of our model amplitude and listed all the parameters on which it depends. We explain here the physical arguments which allow us to qualitatively determine all these parameters.

The overall normalization parameter is easily determined by imposing that the $P$-wave is on top of the unitarity circle at the $\rho$-mass. We have fixed $\sigma_{\infty}$ at $20 \mathrm{mb}$ which seems to be a reasonable value for the asymptotic value of $\pi \pi$ total cross section.

The pomeron parameters have been fixed rather arbitrarily since the physics of the model is essentially contained in the non-pomeron part of the amplitude, and since this pomeron amplitude has been added essentially for the sake of completeness. The parameter $B$ has been chosen in such a way that the slope of the Pomeranchuk trajectory equals 0.5 . With the values chosen for $A, z_{1}$ and $z_{2}$ the pomeron contribution is reasonable at high energy: the $\pi^{+} \pi^{+}$elastic differential cross section (which is supposed to be dominated by the pomeron contribution) turns out to have a behavior very similar to the $\mathrm{K}^{+} \mathrm{p}$ analogue.

The parameters of the meson trajectory function are easily determined by the $\rho$-and $f$-masses and widths. The value of $\alpha_{0}$ seems lower that the one which is currently used, but it is imposed by the parametrization. On the other hand the gmeson appears at a mass rather close to the experimental one.

The determination of the smoothing parameters is more difficult. Remarkably 
(see appendix) the parameter $b$ controls the position of the inelastic thresholds, the slope of the Regge cut, and the scale parameter in the Regge behavior suggesting a new interpretation of the scale factor $s_{0}$, in terms of the average spacing of inelastic thresholds. We have taken $b=0.9 \mathrm{GeV}^{-2}$. With this value the first branch point in $\left(P_{t} C_{s}\right)$ and $\left(C_{t} C_{s}\right)$ is at $s=\left(1-\alpha_{0}\right) / b \approx 0.69 \mathrm{GeV}^{2}$ which does not correspond to any actual two body threshold but is approximately where scattering into four pions begins to become significant. The slope of the effective Regge cut trajectory at $t=0$ is $\frac{1}{2} b=0.45$ as expected from usual absorption models. The scale parameter in the Regge behavior of $\left(P_{t} C_{s}\right)$ is $1 / b \approx 1.1$, which is very close to the canonical value.

All the arguments presented here are qualitative, and in fact it is possible to change the value of $b$ in order to reproduce (for instance) the four-pion threshold. Then one needs to modify the weight function in the $\mu$ integral in such a way that the effects at high energy remain similar (slope of the cut trajectory, scale parameter)

We determine $q$ and $q^{\prime}$ by the following arguments. The $t$-dependence for large fixed $s$ of $\left(P_{s} C_{t}\right)$ is controlled by $q$ and $q$ ' respectively. They both represent Regge cut contributions to the amplitude. We want the Regge cut contributions to resemble Regge cuts obtained in absorption calculation. As such cuts show very little $t$-structure, we tried to find values of $q$ and $q^{\prime}$ which would give us approximately the same $t$-dependence of $\left(P_{s} C_{t}\right)$ and $\left(C_{s} C_{t}\right)$ and so guarantee no structure in the effective cut contribution *. This constraint has essentially determined for us the value of $q / q^{\prime}$, the quantity which is the most sensitive to $q$ turned out to be $\pi^{+} \pi^{-}$total cross section at threshold. We have chosen a value of $q=5$ which corresponds to $\sigma_{\text {th }}=29 \mathrm{mb}$.

We discuss now the determination of the important parameter $\Lambda$. The very nature of the model depends crucially on the value of $\Lambda$. Let $\Lambda_{0}$ be the value of $\Lambda$ for which the contribution of $\left(P_{s} C_{t}\right)$ and $\Lambda\left(C_{S} C_{t}\right)$ cancel approximately at high energy. When $\Lambda=\Lambda_{0}$ one has a model which is approximately a dual crossing symmetric pole-pole model; it is somehow a dualized interference model, essentially the Dolen-HornSchmid interference model [8] where the resonances are described by $\left(P_{s} C_{t}\right)$, the Regge contribution by $\left(P_{t} C_{s}\right)$ and the average of resonances by $-\Lambda_{0}\left(C_{s} C_{t}\right)$. With the form we have chosen $\Lambda_{0}$ is approximately -1 . Now if $\Lambda$ is more negative than $\Lambda_{0}$, then $\Lambda\left(C_{s} C_{t}\right)$ not only cancels at high energy the contribution of $\left(P_{s} C_{t}\right)$ but also reduces the Regge-pole contribution of $\left(P_{t} C_{s}\right)$ and this provides an absorptive correction. One sees then that varying the value of $\Lambda$ allows us to reproduce a very large class of models. One can go from a model which is resonances + background at low energy and Regge pole + Regge cut (cut with the wrong sign) at high energy to a model which is resonance-background at low energy and Regge-pole-Regge-cut at high energy (absorption model-weak or strong absorption according to the strength of $\Lambda$ ), through an ordinary dual model: resonance at low-energy Regge pole at high energy $\left(\Lambda=\Lambda_{0}\right)$. (See table 1.$)$

\footnotetext{
* Of course we cannot have exactly the same $t$ dependence as $\operatorname{Im}\left(C_{S} C_{t}\right)$ shows a zero near $t=-1$, but with the values of $q$ and $q^{\prime}$ which we have chosen the real parts and at least the slopes of the imaginary parts at $t=0$ of $\left(P_{s} C_{t}\right)$ and $\left(C_{s} C_{t}\right)$ fit remarkably well to one another at high energy (see fig. 2).
} 
Table I

\begin{tabular}{|c|c|c|}
\hline $\begin{array}{l}\text { Size of }\left(C_{s} C_{t}\right) \\
\left(\left(P_{S} C_{t}\right)+\Lambda_{0}\left(C_{s} C_{t}\right)=0\right.\end{array}$ & $\begin{array}{l}\text { Low and intermediate } \\
\text { energies }\end{array}$ & Jigh energies \\
\hline$-\Lambda<-\Lambda_{0}$ & $\begin{array}{l}\text { Resonances + background } \\
\text { (double counting) }\end{array}$ & $\begin{array}{l}\text { Regge poles + Regge cuts } \\
\text { (wrong sign of the cut } \\
\text { correction) }\end{array}$ \\
\hline$\Lambda=\Lambda_{0}$ & $\begin{array}{l}\text { Dolen-florn-Schmid } \\
\text { interterence model: } \\
\text { resonances + Regge } \\
\text { resonances }\end{array}$ & Pure Regge-pole model \\
\hline$-\Lambda>-\Lambda_{0}$ & $\begin{array}{l}\text { Resonances - inelasticity } \\
\text { (absorption of interior } \\
\text { resonances) }\end{array}$ & $\begin{array}{l}\text { Regge poles .... Regge cuts } \\
\text { (absorption model) }\end{array}$ \\
\hline
\end{tabular}

Thus we see that the proper to deal with the problem of double counting is to subtract a contribution larger than the average of the resonances so that one has an absorptive correction at all energies.

Thus the determination of $\Lambda$ is an essential point in the physics of the model. Since the amplitude is linear in $\Lambda$, it is clear that we can fix it by imposing one zero on the amplitude, at a single value of $s$ and a single value of $t$. The two most dramatic zeros in conventional amplitudes are the Adler zero, with the amplitude vanishing at $s=t=u=m_{\pi}^{2}$ (where it is real); and the crossover zero which arises from the imaginary part of the $\rho$-exchange amplitude vanishing near $-t=0.2$ at large $s$.

The pomeron contribution we use vanishes automatically at the Adler point; the non pomeron part must also vanish there if the amplitude is to satisfy the Adler consistency condition. This is the condition we have used to determine $\Lambda$.

To summarize, we have now written an explicit resonance - Regge amplitude which has resonances at low energies dual to absorbed Regge poles (i.e. Regge poles-cuts) at high energies, and satisfying our initial requirements. It depends on the input trajectory plus six parameters ( $a, b, q, q^{\prime}, \Lambda$ and overall scale). Three of these parameters are determined from general arguments $(a=0$ to have the threshold cuts extend to infinity, $q^{\prime} / q$ to have various terms with the same $t$-dependence, and $\Lambda$ from the Adler condition) and the rest from low-energy conditions (the value of $\sigma_{\mathrm{T}}$ at threshold and at the $\rho$, and the location of the first inelastic threshold).

We can now examine the amplitude at all energies and angles (apart from the high-energy large-angle region where there is an asymptotic blowup because we have used a smoothed Veneziano kernel). In particular, the entire high-energy behavior of the amplitude is determined, and its remarkable agreement with our expectations is very satisfying.

\subsection{Discussion of the results}

Figs. 1 to 17 show the results obtained for $\pi \pi$ scattering with the values of the parameters shown in table Al. 


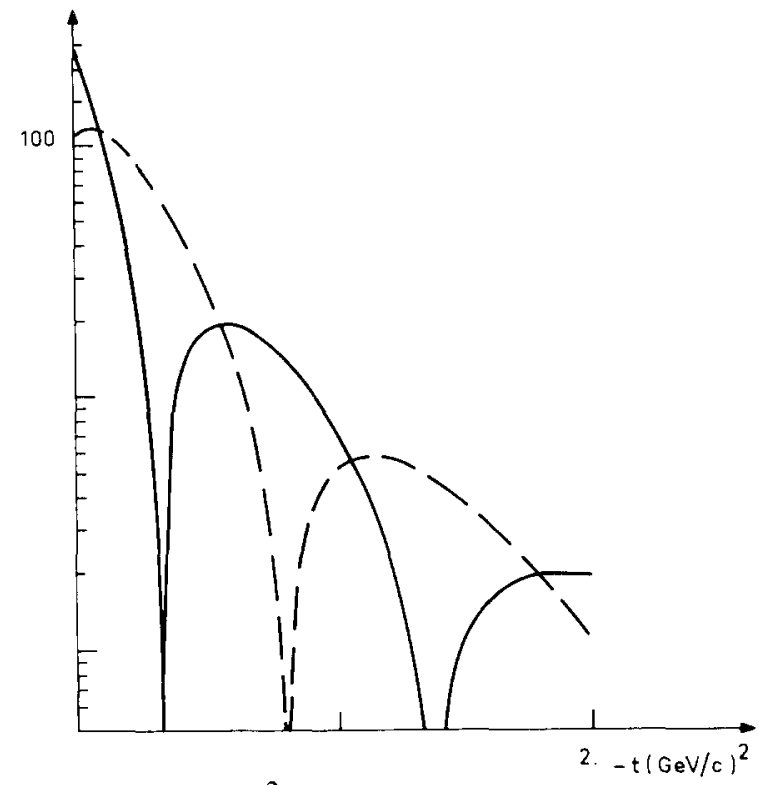

Fig. 1. The $\left(P_{t} C_{s}\right)$ term at $s=50 \mathrm{GeV}^{2}$. The full line is $\log \mid$ imaginary part $\mid$ and the dashed line is $\log \mid$ real part $\mid$. This shows the Regge phase of $\left(P_{t} C_{S}\right)$.

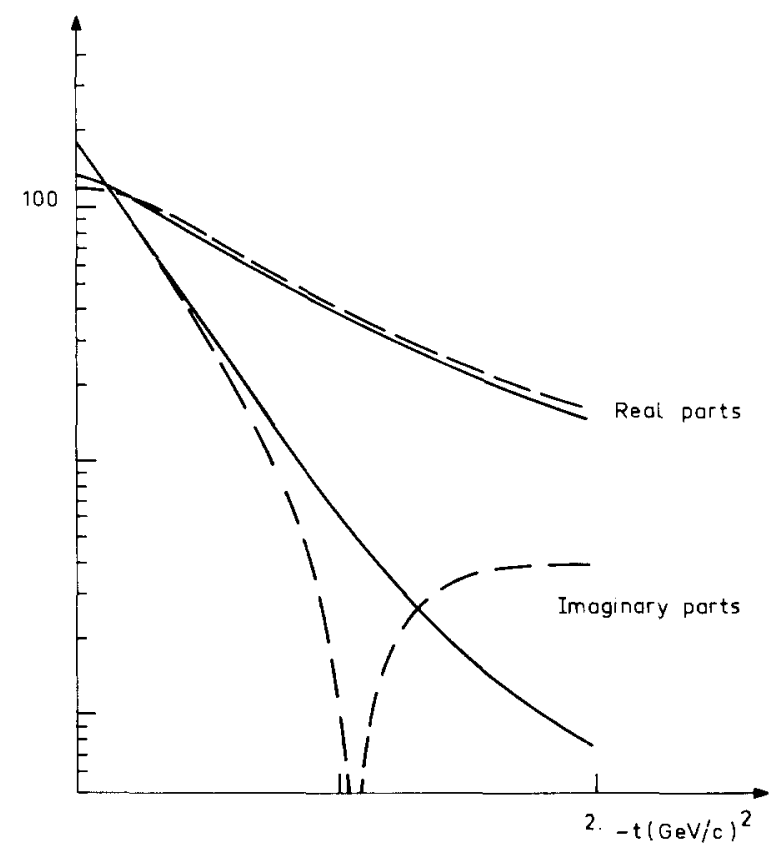

Fig. 2. Comparison of $\left(P_{s} C_{t}\right)$ (full lines) and $\left(C_{s} C_{t}\right)$ (dashed lines), normalized to have the same imaginary parts at $t=0\left(\Lambda=-\Lambda_{\mathrm{o}}\right)$, and $s=50 \mathrm{GeV}^{2}$, to illustrate the similarity of their $t$-dependence. 

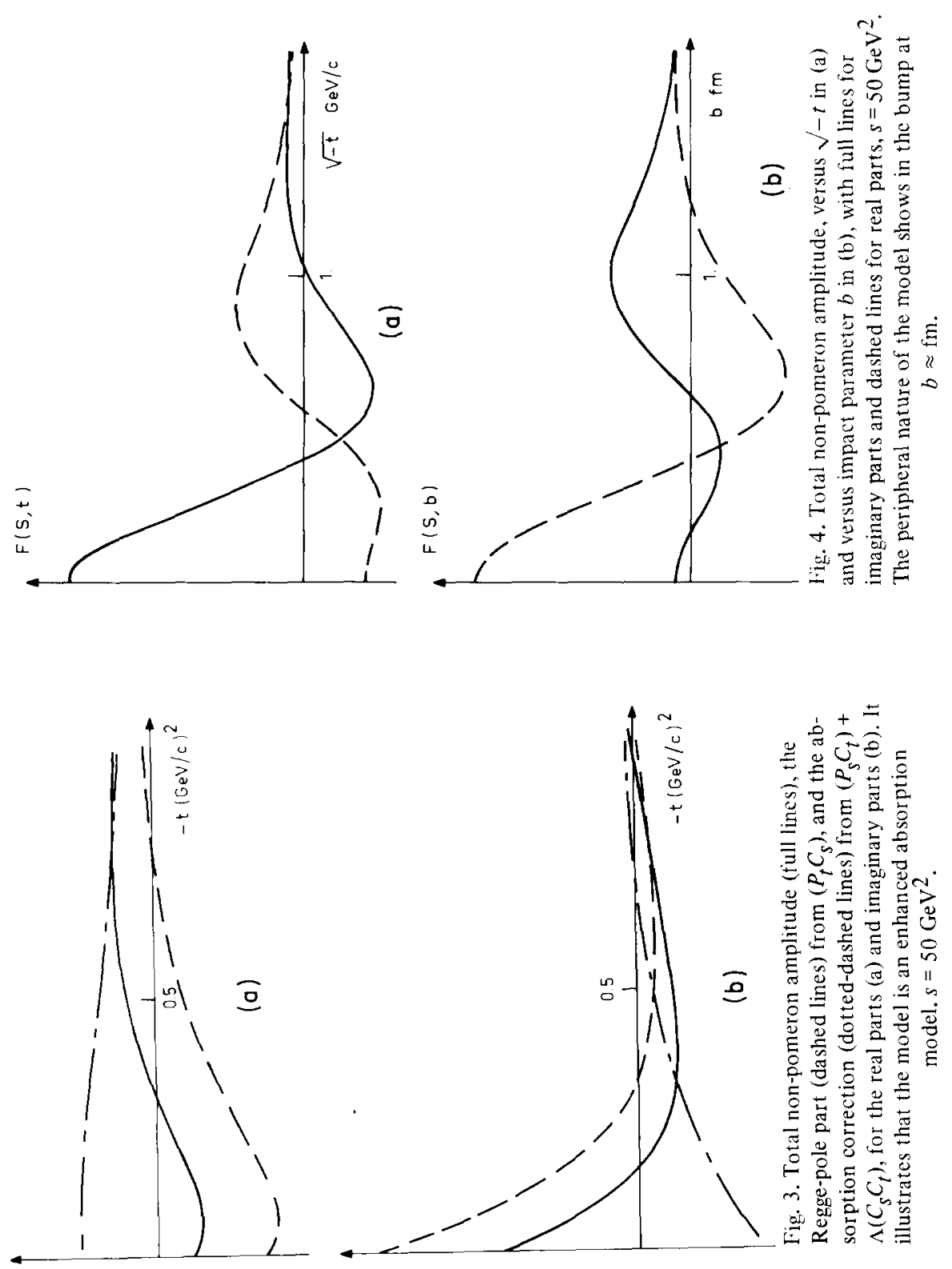


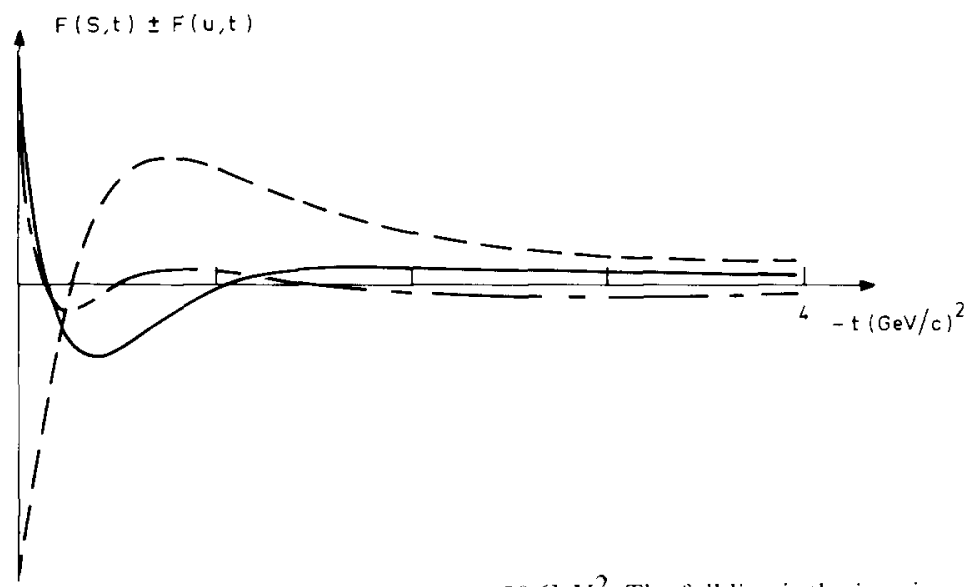

Fig. 5. The signatured amplitudes versus $t$ at $s=50 \mathrm{GeV}^{2}$. The full line is the imaginary part. common to both signatures (strong exchange degeneracy). The dashed line is the positive signature real part $\left(\mathrm{P}^{\prime}+\mathrm{P}^{\prime} * \mathrm{P}\right.$ cut $)$, and dotted-dashed line the negative signature real part $(\rho+\rho * \mathrm{P}$ cut $)$

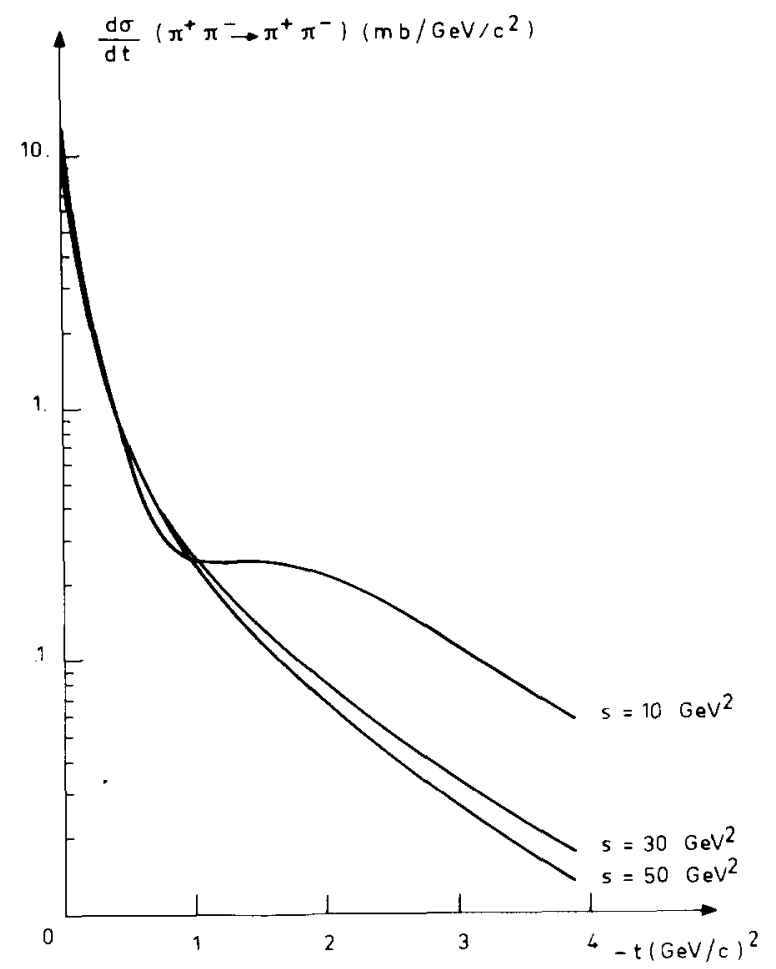

Fig. 6. $\pi^{+} \pi^{-}$elastic differential cross section near the forward direction at a few energies. 

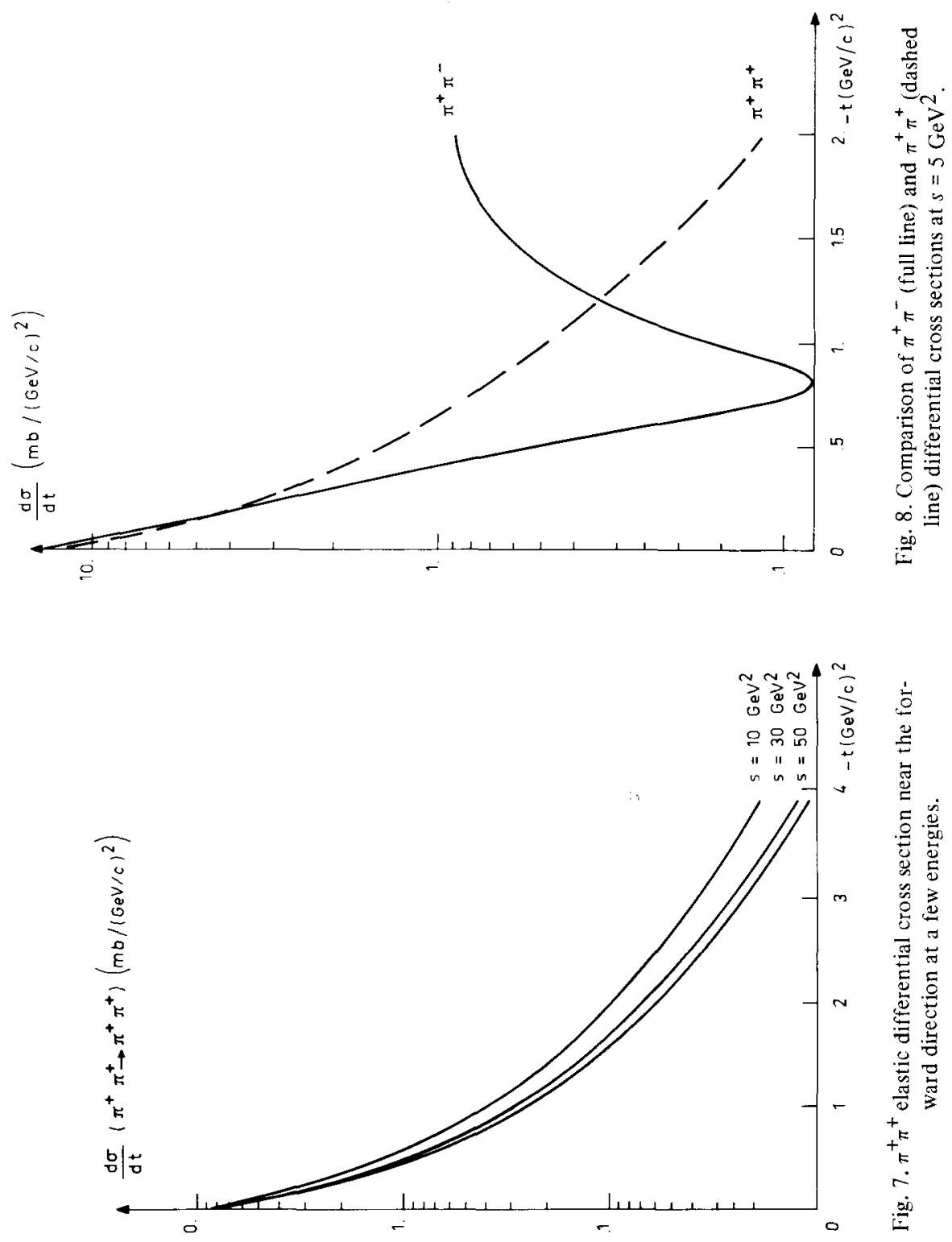

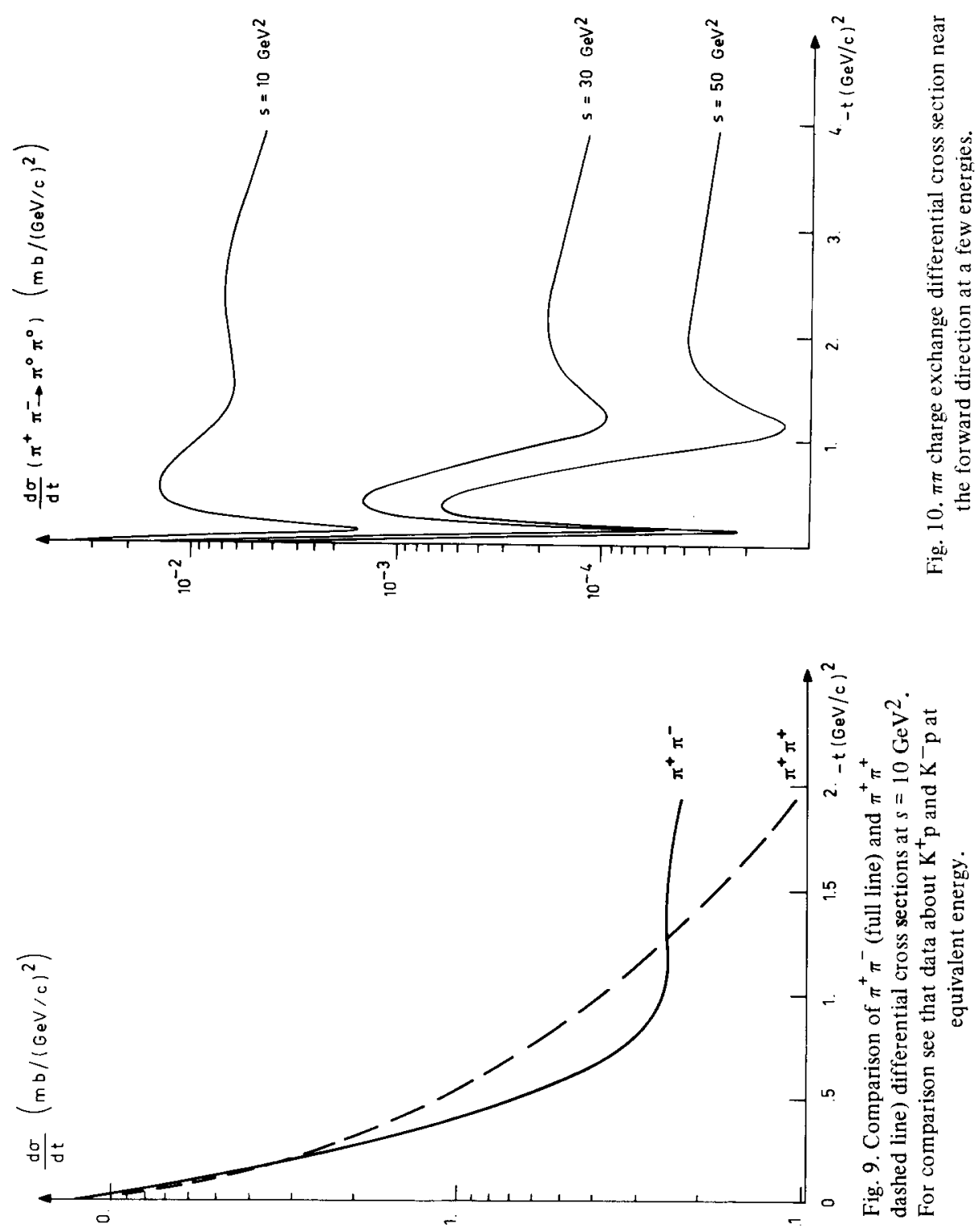

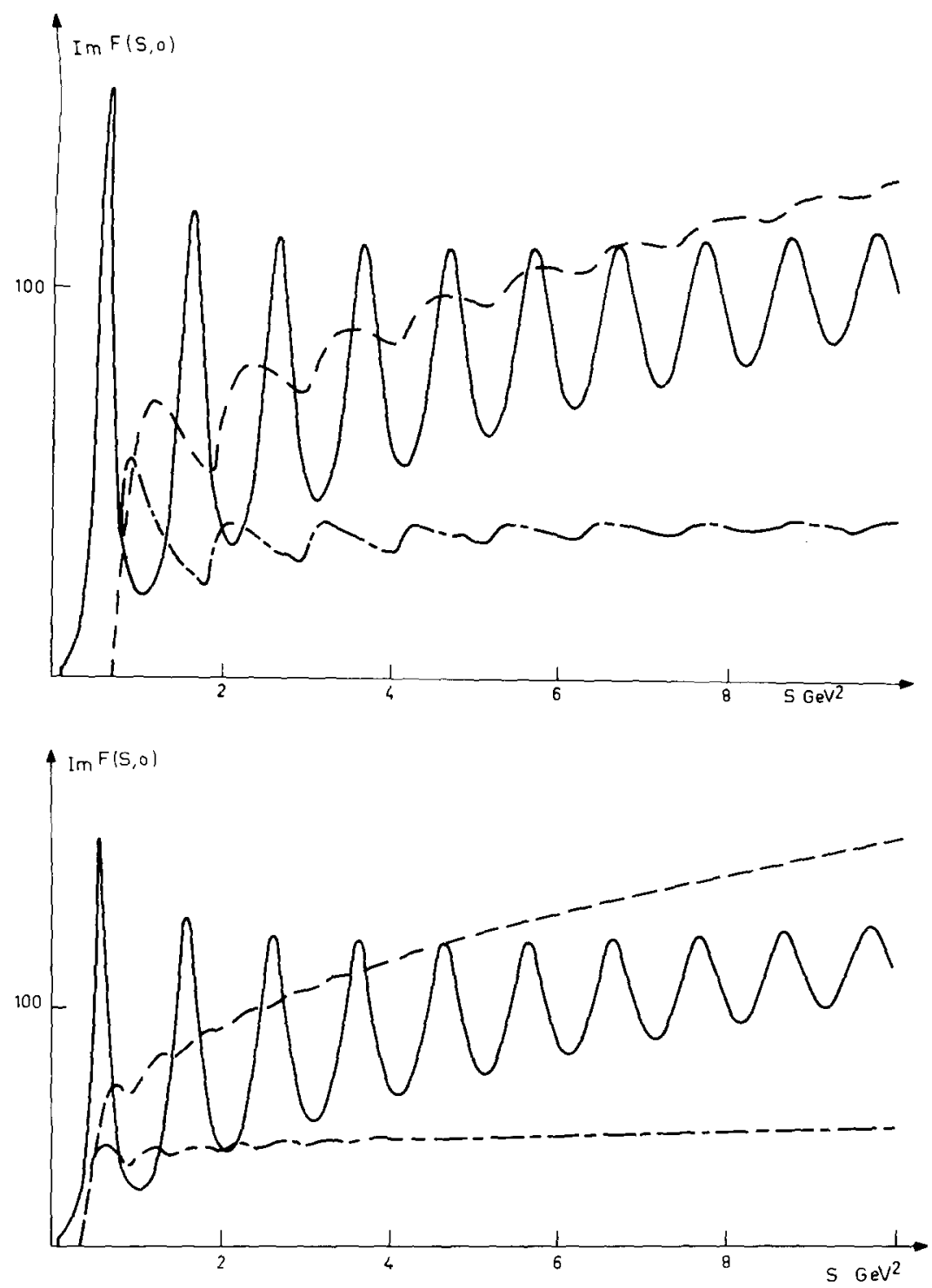

Fig. 11. Analysis of the non-pomeron, forward direction imaginary part. The full line is the resonance contribution from $\left(P_{s} C_{t}\right)$, the dashed line, the Regge-pole contribution from $\left(P_{t} C_{s}\right)$. The dotted-dashed line represents the quantity one has to subtract from the resonance contribution in order to get the final answer. We call this term the absorption of the resonances: it is equal to $-\left(P_{t} C_{s}\right)-\Lambda\left(C_{s} C_{t}\right)$. Fig. 11 a is obtained with the parametrization of eqs. (5) and (6) whereas fig. $1.1 \mathrm{~b}$ correspond to the modification suggested in appendix. 


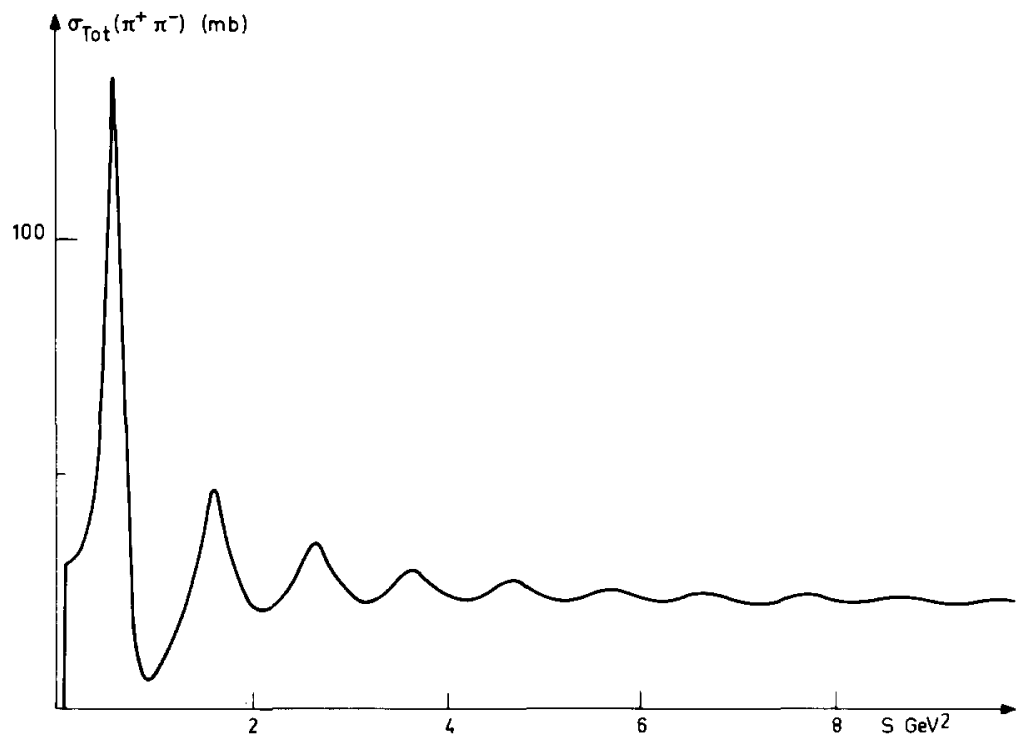

Fig. $12 . \pi^{+} \pi^{-}$total cross section from threshold to $10 \mathrm{GeV}^{2}$.

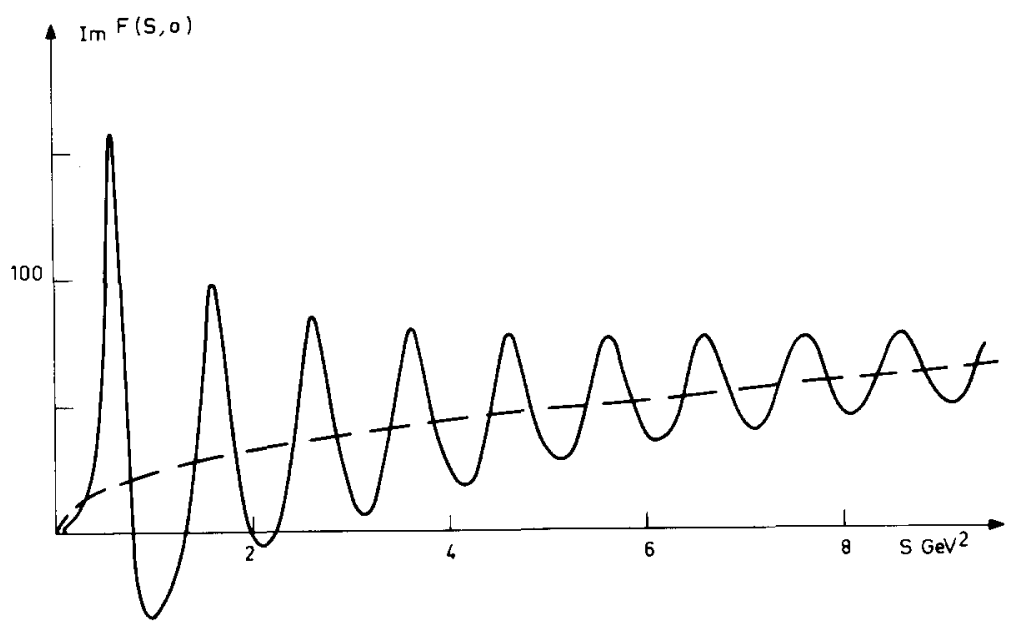

Fig. 13. Global duality test: comparison of the forward imaginary part of the non-pomeron amplitude (full line) with the extrapolation at low energy of the high-energy approximation (dashed line). 


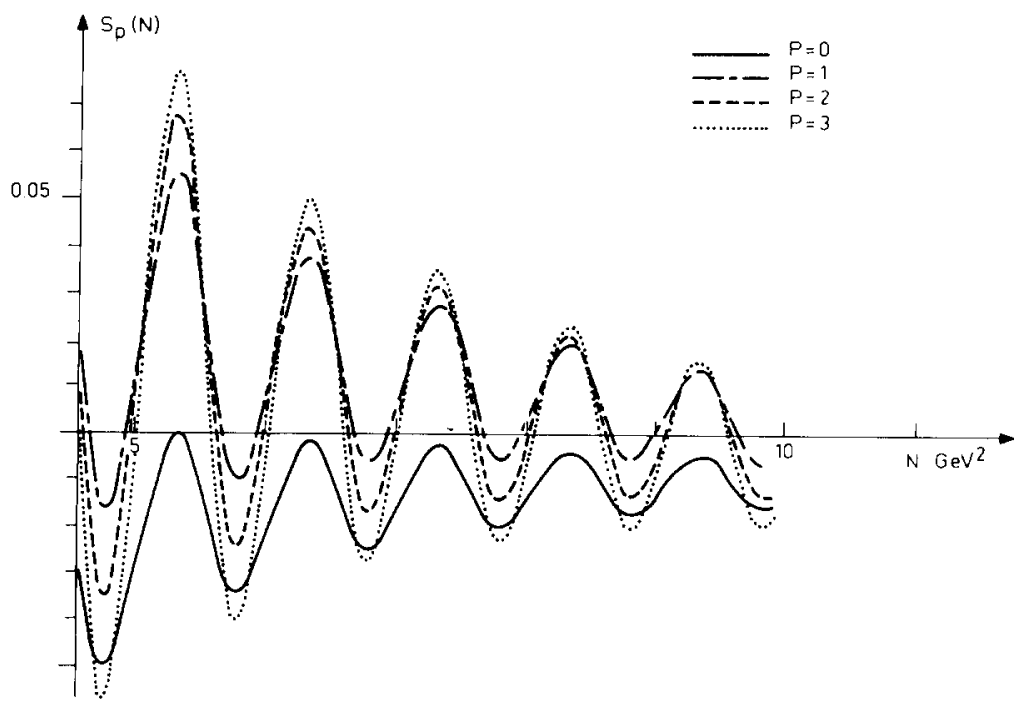

Fig. 14. Numerical test of finite energy sume rules.

$$
S_{p}(N)=\frac{\int_{4 m_{\pi}^{2}}^{N} s^{p}\left[\operatorname{Im} F(s, 0)-\operatorname{Im} F_{\text {asympt. }}(s, 0)\right] \mathrm{d} s}{\int_{4 m_{\pi}^{2}}^{N} s^{\beta}\left[\operatorname{Im} F(s, 0)+\operatorname{Im} F_{\text {asympt. }}(s, 0)\right] \mathrm{d} s} .
$$

Plot of $S_{p}(N)$ versus $N$ for $p=0,1,2,3$.

Figs. 1 to 10 show the high energy results; as a typical high-energy value we have taken $s=50 \mathrm{GeV}^{2}$. Actually, the asymptotic regime is reached at a much lower energy of the order of $20 \mathrm{GeV}^{2}$. The plot of $\operatorname{Re}\left(P_{t} C_{s}\right)$ and $\operatorname{Im}\left(P_{t} C_{s}\right)$ versus $t$ in fig. 1 clearly snows the expected Regge phase corresponding to two exchange degenerate trajectories $\left(\mathrm{e}^{-i \pi \alpha(t)}\right)$. Fig. 2 shows a comparison of the $t$-dependences of the two terms $\left(P_{s} C_{t}\right)$ and $\left(C_{s} C_{t}\right)$ which exhibit the Regge cut behavior. When the imaginary parts are normalized at $t=0\left(\Lambda=-\Lambda_{0}\right)$ the real parts match fairly well whereas the imaginary parts desagree at large $t$ but have essentially the same slope at $t=0$.

Figs. 3-5 show that with the value of $\Lambda$ determined by the Adler self consistency condition our model is an enhanced absorption model. The pole-cut destructive interference which produces the diffractive zeros is shown in fig. 3 . The peripheral nature of the imaginary part is exhibited by the Hankel transform, fig. 4 , which shows a bump near $b \approx 1 \mathrm{fm}$. Finally the resulting amplitudes of both signatures are shown in fig. 5 . 


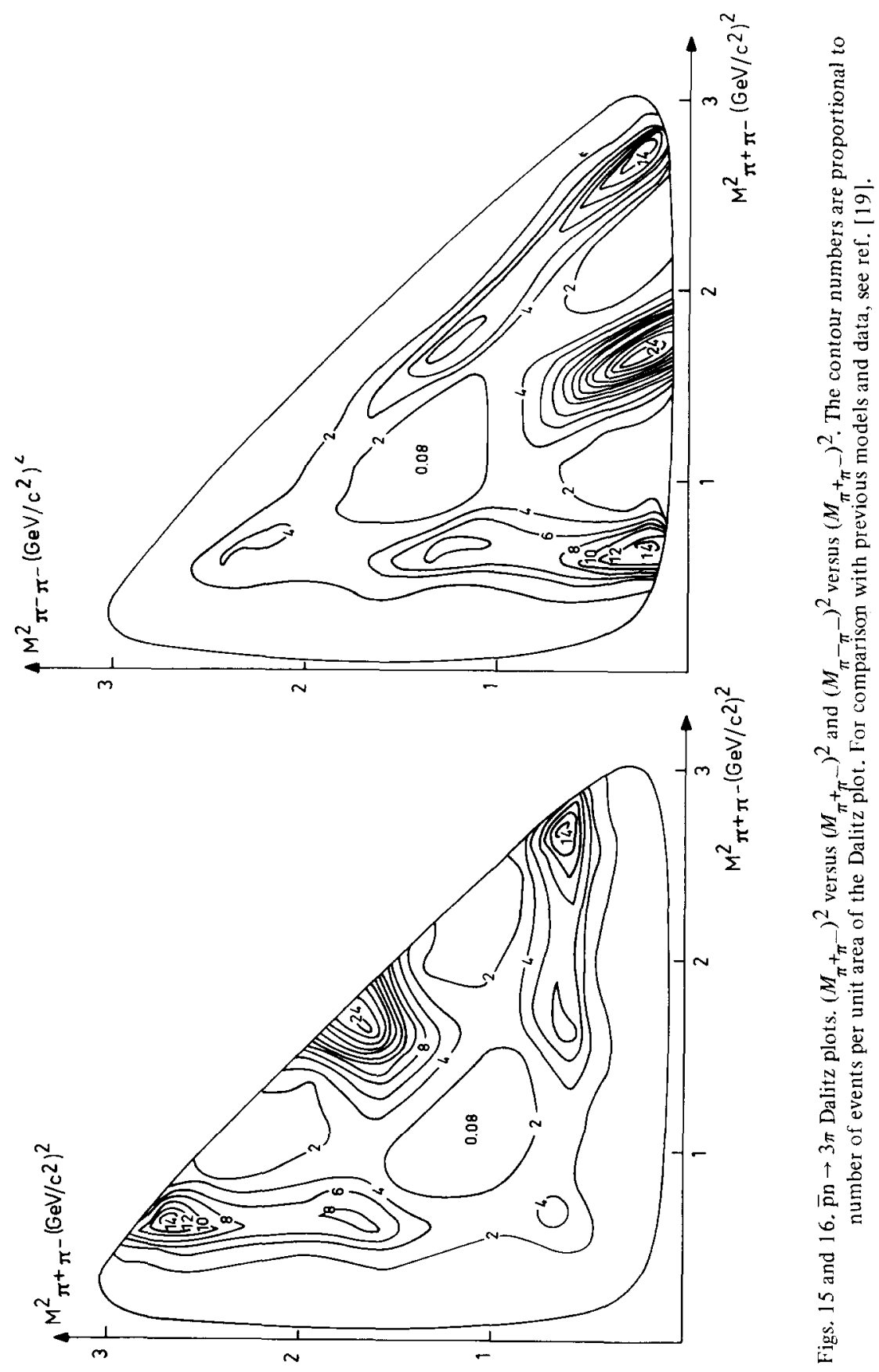




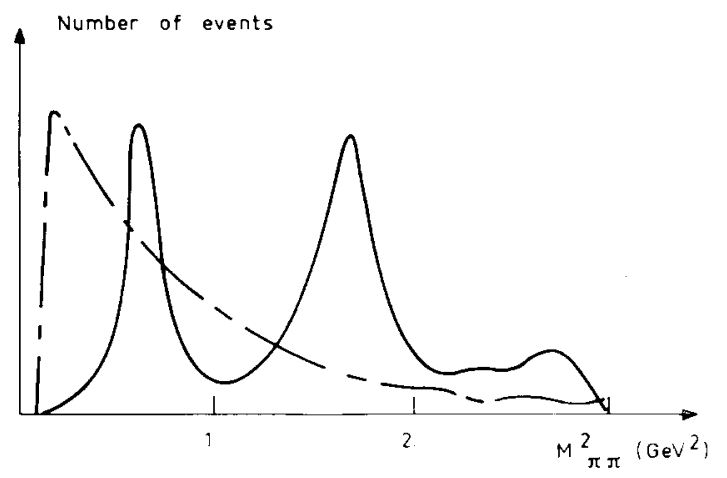

Fig. 17. Integrated $\pi^{+} \pi^{--}$(full line) and $\pi^{-} \pi^{-}$(dashed line) spectra in $\overline{\mathrm{p}} \mathrm{n} \rightarrow \pi^{+} \pi^{-} \pi^{-}$.

We want to emphasize the fact that the absorption effects which are easy to obtain in a standard model where cut corrections are somehow derived from unitarity, are reproduced here via a completely different approach based on duality and crossing symmetry. Since, in our knowledge there did not exist up to now any crossing symmetric absorption model we think that our results show at least that enhanced absorption (people familiar with absorption techniques will recognize how close to enhanced absorption [3] our amplitude is) is not incompatible with rigorous crossing symmetry at all energies.

We have not been able to follow the Adler zero as a function of $s$ (in particular in the resonance region ) but we conjecture that it becomes at intermediate energy the dual zero in the $t$-dependence at the resonances, and at high energies the crossover zero. This conjecture is based on the remark that all these zeros do have, in our model, the same origin, that is cancellations between different components of the amplitude. In this respect our model differs drastically from the Veneziano Lovelace model in which the Adler zero moves along a straight line $s+t=$ constant and each zero in the residue of a resonance comes from a different line $s+t=$ constant, even though they appear at the same $t$-values $[17,18]$. The amplitude of our model, on the other hand, has one zero which is the Adler zero, the zero in all resonance residues at $t \approx 0.2$, and the high-energy absorptive zero.

Some typical intermediate and high-energy differential cross sections are shown in figs. 6 to 10 . The most spectacular effect is the comparison of the exotic cross section $\left(\pi^{+} \pi^{+}\right)$and the non exotic one $\left(\pi^{+} \pi^{-}\right)$(figs. 8 and 9$)$ showing the two crossovers, the structure in the non exotic cross section and the lack of structure in the exotic one. Figs. 8 and 9 should be compared with the data of $\mathrm{K}^{+} \mathrm{p}$ and $\mathrm{K}^{-} \mathrm{p}$ elastic scattering.

Low-energy results are shown in figs. $11 \mathrm{a}, 11 \mathrm{~b}$ and 12 . Figs. $11 \mathrm{a}$ and $11 \mathrm{~b}$ show (for two choices of parameters) the decomposition of the imaginary part of the non pomeron amplitde at $t=0$. The full line curve is the contribution of the $\left(P_{s} C_{t}\right)$ term (resonances). The dashed curve represents the contribution of $\left(P_{t} C_{s}\right)$ (Regge 
pole). It is clear in this figure that the model with $\Lambda=0$ would be a bad interference model; the resonance signals would hardly emerge on the Regge background. On the contrary, with the actual value of $\Lambda$, one has to subtract the dotted-dashed curve $\left(-C_{s} P_{t}-\Lambda C_{s} C_{t}\right)$ from the resonance contribution in order to get the final answer. It is of the form resonance minus absorption. Notice that the dotted-dashed curve which can be interpreted as an inelasticity does vary very rapidly in the resonance region.

It is interesting to study the partial wave content of the total absorption of the resonance: between the first two thresholds only the $\mathrm{S}$ - and $\mathrm{P}$-waves are absorbed, so in the second resonance peak the $\mathrm{f}_{\mathrm{o}}$ (D-wave) is not affected by absorption whereas the $\rho^{\prime}$ (P-wave) is. When all these corrections are applied, one might think that the shape of the resonances would be distorted. This is not the case as can be seen in fig. 12 which shows the total $\pi^{+} \pi^{-}$cross section up to $s=10 \mathrm{GeV}^{2}$ and in fig. 13 which shows the imaginary part at $t=0$ of the total non pomeron amplitude compared to the extrapolation to low energy of the high energy approximation (Reggepole-Regge-cut obtained through at fit at $s=50 \mathrm{GeV}^{2}$ and $s=100 \mathrm{GeV}^{2}$ to the amplitude of the model). Finite energy sum rules are tested on fig. 14.

At the $\rho$-mass, the $\mathrm{S}$-wave is also resonating (the $\sigma$-meson). We find at this point that the $S$-wave badly violates unitarity, being too large approximately by a factor of three. A change in the parameters caused almost no change in this unitarity violation. It appears that the existence of the Adler zero is inconsistent with unitarity if the mass and width of the $\sigma$ are the same as those of the $\rho$, as must occur if the Veneziano kernel is used.

Our last result concerns the three pion decay of the $\bar{p}$ system at rest. Since the $\overline{\mathrm{p}} \mathrm{n}$ system at rest has the quantum numbers of a $\pi^{-}$, we can assume, as proposed by Lovelace [14], that the decay amplitude can be obtained (up to an unknown overall normalization) by analytic continuation in $s, t, u$ of the $\pi^{+} \pi^{-}$elastic amplitude from the scattering region to the decay one. Since our numerical integration technique also works when both $s$ and $t$ are positive we have been able to perform this analytic continuation explicitly. The results are shown in figs. 15 to 17. We want to stress that we have not changed anything in the model while going from the scattering to the decay region. In some sense our result about the $\bar{p}$ n decay has to be considered as a "prediction" since the amplitude was "fitted" from the $\pi^{+} \pi^{-} \rightarrow \pi^{+} \pi^{-}$ data. Qualitatively the results are fairly good, and actually comparable with any of the several previous models which were constructed to apply only to the annihilation data [19]. Although the peaks are too narrow, the dip-bump structure is well reproduced. Angular distributions show that the $\sigma$ dominates the $\rho$, but no interesting conclusion can be drawn from this fact since it occurs also in the scattering region, where it has to be considered as a defect. We insist on the fact that our model is not intended to reproduce the $\bar{p} n$ annihilation data. Applying our model to this process is just a way to explore the properties of our $\pi \pi$ scattering amplitude in the unphysical region where both $s$ and $t$ are positive. 


\section{DISCUSSION AND REMARKS}

In our view, we have constructed a model for a hadron scattering amplitude which gives a good description of experimental data, at a level which is almost quantitative, at all energies and angles. We are encouraged to believe that the ideas we have incorporated (analyticity, crossing, Regge behavior, duality, and partial unitarity via requiring second sheet resonance poles at low energies and absorption effects at high energies) are important ingredients in full description of experimental data.

In view of the fact that our amplitude is slightly more complicated than a simple Veneziano one it may be useful to make a few remarks about the significance of the complexity. On the one hand, the Veneziano model is simple, but it does not look like experimental data. On the other hand, we have introduced various complications related to unitarity effects, but our amplitude might be very much like the experimental data. In addition, we have good qualitative agreement with data in all regions (including $\overline{\mathrm{p}} \mathrm{n} \rightarrow 3 \pi$ ) simultaneously, without arbitrary modifications such as unitarization in one energy range, absorption in another, etc., and we never give up nice properties such as crossing, Regge behavior, proper spin content, etc. Thus we feel that the kind of complications we have are more or less the minimal amount necessary to get good agreement with data. As we gain experience with such models it seems likely that they will grow simpler and easier to understand.

To test these ideas and models such as ours further, we need to make progress in two directions. First, we want to remove the problems associated with the Veneziano kernel, such as the fixed angle divergence, and the too large S-wave beneath the $\rho$. It appears to us that using a Virasoro kernel [20] would be suitab]e for this purpose: the Virasoro ansatz puts the three crossed channels on the same footing, since, with ou smoothing procedure we know how to transform poles into cuts, we can build terms with, for instance poles in one channel and cuts in the two other (in particular cuts similar to the Regge-Regge cuts in the exotic channel). Since, now the behavior in all directions is governed by Regge singularities, we are sure that no blow up occurs in the full angular distribution, from forward to backward directions. On the other hand, since exchange degeneracy is not rigidly imposed, much more flexibility is available to adjust the $\sigma$ to $\rho$ ratio. One of us (R.L.) is pushing investigations in this direction. Secondly, recent progresses made in dealing with $s-x$ dual amplitudes [12] put within our possibilities the performing of what we consider to be the best absorption dual model (see eq. (1)).

In order to further test our ideas, it is necessary to write similar amplitudes for general reactions, including those with spin. It is not entirely clear how to proceed, because one wants to write expressions such as the one we have used for amplitudes with simple crossing properties. But to compare with the absorption ideas at high energies one wants amplitude with definite net helicity flip in the appropriate channels. It does not appear that any basic difficulties arise in going to a process such as $\pi^{-} \mathrm{p} \rightarrow \pi^{\circ} \mathrm{n}$, and we are beginning to carry this out. 
It should be noted that we can more easily check one aspect of the spin dependence by considering $\pi \pi \rightarrow \pi \omega$. This process still has only one amplitude, but it has net helicity flip one. Thus we expect $\operatorname{Im} M \approx 0$ at $-t=0.6$ at all energies, rather than 0.2 for $n=0$ amplitudes as in $\pi \pi$. This effect should arise naturally; it does at high energies in a real absorptive cut model because the cut discontinuity has an extra power of $\sqrt{ }-t$ and the cut is correspondingly smaller. Here the integrand weight functions will reflect this, with perhaps a factor $\lambda^{\frac{1}{2 n}}$ in the in tegrand being suggested for amplitudes of different net helicity flip $n$. At low energies it will be automatic if the peripheral resonances dominate, since the appropriate partial wave expansion is in terms of $d_{10}^{J}(\theta)$, which has a forward zero and then one at $\cos \theta=\frac{1}{5}$. Using the peripheral relation between $J$ and $s$ gives a zero at $-t \approx 0.6$.

Another aspect of interpretation which needs more detailed study is the precise connection to the absorption model. Several directions could be pursued. One could study the $J$-plane structure of a typical absorption model and of our amplitude and make them as alike as possible. Or, one could try to use duality and low-energy constraints to get at the absorption model. One can also see how duality and crossing effects modify asymptotic absorption model predictions at a finite energy. The relative success of our model, which is an investigation in a new field of research, shows that the key of all these opened questions lies, may be, in a systematic study at all energies, of the implications of crossing, unitarity, duality and absorption.

\section{APPENDIX. PARAMETRIZATION AND MATHEMATICAL PROPERTIES OF THE AMPLITUDES}

$A(s, t)$, the $\pi^{+} \pi^{-} \rightarrow \pi^{+} \pi^{-}$elastic scattering amplitude is written as

$$
A(s, t)=\sigma_{\infty} P(s, t)+\gamma F(s, t)
$$

where $P(s, t)$ is the pomeron amplitude and $F(s, t)$ the non pomeron amplitude.

\section{The pomeron amplitude $P(s, t)$}

Crossing symmetry

$$
\begin{aligned}
& P(s, t)=H(s, t)+H(t, s), \\
& H(s, t)=H(u, t) .
\end{aligned}
$$

Parametrization (see table A.l for the values of parameters)

$$
H(s, t)=-\frac{1}{\pi}(s-u) f(t)\left(\frac{\left[\sqrt{4 m_{\pi}^{2}-s+A}\right]^{2\left(\alpha_{\mathrm{p}}(t)-1\right)}-\left[\sqrt{4 m_{\pi}^{2}-u}+A\right]^{2\left(\alpha_{\mathrm{p}}(t)-1\right)}}{2\left(\alpha_{\mathrm{p}}(t)-1\right)}\right),
$$


with

$$
\begin{aligned}
& \alpha_{\mathrm{p}}(t)=1-\left(\sqrt{4 m_{\pi}^{2}-t}-2 m_{\pi}\right) /\left(\sqrt{4 m_{\pi}^{2}-t}+B\right) \\
& f(t)=\exp \left(-\left(\frac{z_{1}-t}{z_{2}}\right)^{\frac{1}{4}}+\left(\frac{z_{1}}{z_{2}}\right)^{\frac{1}{4}}\right) .
\end{aligned}
$$

Table A.1

\begin{tabular}{|c|c|c|c|}
\hline $\begin{array}{l}\text { trajectory function } \\
\text { smoothing parameters } \\
\text { absorption parameter } \\
\text { pomeron trajectory } \\
\text { pomeron residue } \\
\text { normalizations }\end{array}$ & $\begin{aligned} & \alpha_{0}=0.375 \\
& b=0.9 \mathrm{GeV}^{-2} \\
& A=0.2 \mathrm{GeV} \\
& \gamma=-\end{aligned}$ & $\begin{aligned} & \alpha^{\prime}=0.985 \mathrm{GeV}^{-2} \\
& q=5.0 \\
& \Lambda=-1.89 \\
& B=3.5 \mathrm{GeV} \\
& z_{1}=4 m^{2} \\
& 5 \mathrm{GeV}^{4}\end{aligned}$ & $\begin{aligned} g & =0.137 \mathrm{GeV}^{-1} \\
q^{\prime} & =7.0\end{aligned}$ \\
\hline
\end{tabular}

Parameters of the model

\begin{tabular}{|c|c|c|c|}
\hline & $\left(P_{s} C_{t}\right)(s, t)$ & $\left(P_{t} C_{s}\right)(s, t)$ & $\left(C_{s} C_{t}\right)(s, t)$ \\
\hline \multirow[t]{5}{*}{$\begin{array}{l}\text { Analyticity in } s \\
\text { for fixed } t\end{array}$} & $\begin{array}{l}\text { Real analytic with } \\
\text { a cut from } \\
s=4 m_{\pi}^{2} \text { to infinity }\end{array}$ & Real analytic & Real analytic \\
\hline & \multirow[t]{2}{*}{$\begin{array}{l}\text { Second sheet poles } \\
\text { at } s=s_{n} \text { such that } \\
\alpha\left(s_{n}\right)=n=1,2,3, \ldots\end{array}$} & $\begin{array}{l}\text { Branch cuts from } \\
s_{n}^{c}=\frac{-\left(\alpha_{0}-1\right)+n}{b}\end{array}$ & $\begin{array}{l}\text { Branch cuts from } \\
s_{n}^{c}=\frac{-\left(\alpha_{0}-1\right)+n}{b}\end{array}$ \\
\hline & & $\begin{array}{l}\text { to infinity; } \\
n=0,1,2, \ldots\end{array}$ & $\begin{array}{l}\text { to infinity; } \\
n=0,1,2, \ldots\end{array}$ \\
\hline & \multirow[t]{2}{*}{$\begin{array}{l}\text { Residue of pole } \\
s_{n} \text { : polynomial in } \\
t \text { of degree } n\end{array}$} & $\begin{array}{l}\text { Discontinuity } \\
\text { between } s_{n}^{c} \text { and } \\
c_{n+1}^{c}: \\
\text { poly nomial in } \\
\alpha(t) \text { of degree } n\end{array}$ & $\begin{array}{l}\text { Discontinuity } \\
\text { between } s_{n}^{c} \text { and } \\
s_{n+1}^{c}: \\
\text { polynomial in } \\
t \text { of degree } n\end{array}$ \\
\hline & & $\begin{array}{l}\text { Behavior near } \\
s_{n}^{c}:\left(s_{n}^{\mathrm{c}}-s\right)^{\frac{3}{2}}\end{array}$ & $\begin{array}{l}\text { Behavior near } \\
s_{n}^{c}:\left(s_{n}^{c}-s\right)^{\frac{3}{2}}\end{array}$ \\
\hline $\begin{array}{l}\text { Analyticity in } t \\
\text { for fixed } s \geqslant 4 m_{\pi}^{2}\end{array}$ & $\begin{array}{l}\text { Holomorphic for } \\
t<s_{\mathrm{O}}^{c}\end{array}$ & $\begin{array}{l}\text { Holomgrphic for } \\
t<4 m_{\pi}^{2}\end{array}$ & $\begin{array}{l}\text { Holomorphic for } \\
t<s_{\mathrm{O}}^{c}\end{array}$ \\
\hline \multirow[t]{3}{*}{ High-s behavior } & $\begin{array}{l}\text { Regge cut } \\
\text { behavior }\end{array}$ & $\begin{array}{l}\text { Regge pole } \\
\text { behavior }\end{array}$ & $\begin{array}{l}\text { Regge } c u t \\
\text { behavior }\end{array}$ \\
\hline & $(-s)^{\alpha_{\mathrm{c}}(t)}$ & \multirow[t]{2}{*}{$(-s)^{\alpha(t)}$} & 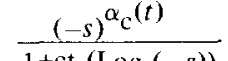 \\
\hline & $1+\mathrm{ct}(\log (-s))$ & & $1+\mathrm{ct}(\log (-s))$ \\
\hline
\end{tabular}

Table A.2 
2. The non-pomeron amplitude $F(s, t)$

$$
F(s, t)=\left(P_{s} C_{t}\right)(s, t)+\left(P_{t} C_{s}\right)(s, t)+\Lambda\left(C_{s} C_{t}\right)(s, t)
$$

\section{Crossing symmetry}

$$
\left(P_{t} C_{s}\right)(s, t)=\left(P_{s} C_{t}\right)(t, s), \quad\left(C_{s} C_{t}\right)(s, t)=\left(C_{s} C_{t}\right)(t, s) .
$$

Parametrization (see table A.1 for the values of the parameters)

$$
\begin{gathered}
\left(P_{s} C_{t}\right)=\int_{0}^{\infty} \mathrm{d} \lambda \int_{0}^{b} \mathrm{~d} \mu \mathrm{e}^{-\lambda} \sqrt{\mu(b-\mu)} \frac{\Gamma(1-\alpha(s)) \Gamma\left(-\left(\alpha_{0}-1\right)(1+\lambda / q)-\mu t\right)}{\Gamma\left(-\alpha(s)-\left(\alpha_{0}-1\right)(1+\lambda / q)-\mu t\right)} \\
\left(C_{s} C_{f}\right)=\int_{0}^{\infty} \mathrm{d} \lambda \int_{0}^{b} \mathrm{~d} \mu \mathrm{e}^{-\lambda} \sqrt{\mu(b-\mu)} \frac{\Gamma\left(-\left(\alpha_{0}-1\right)\left(1+\lambda / q^{\prime}\right)-\mu s\right) \Gamma\left(-\left(\alpha_{0}-1\right)\left(1+\lambda / q^{\prime}\right)-\mu t\right)}{\Gamma\left(-2\left(\alpha_{0}-1\right)\left(1+\lambda / q^{\prime}\right)-\mu s-\mu t-1\right)} \\
\alpha(s)=\alpha_{0}+\alpha^{\prime} s-g\left[\sqrt{4 m_{\pi}^{2}-s}-2 m_{\pi}\right]
\end{gathered}
$$

The mathematical properties are summarized in table A.2.

\section{Derivation of the mathematical properties}

We have studied extensively the analyticity properties (residues of poles and discontinuities of cuts) and the asymptotic behaviours.

We use the following notations and properties:

$C_{k}^{m}$ the binomial coefficients

$D_{k}^{m}$ the coefficients of the Pochhammer polynomial expansion

$$
(x-m)_{m}=(x-1) \ldots(x-m)=\frac{\Gamma(x)}{\Gamma(x-m)}=\sum_{k=0}^{m} D_{k}^{m} x^{k},
$$

$\phi(a, c, x)$ the degenerate hypergeometric series [21]

$$
\begin{aligned}
& \phi(a, c, x)=\sum_{n=0}^{\infty} \frac{(a)_{n} x^{n}}{(c)_{n} n !}, \\
& \phi\left({ }_{2}^{3}, 3, x\right)=\mathrm{e}^{\frac{1}{2} x} \sum_{n=0}^{\infty} \frac{\left(\frac{1}{16} x^{2}\right)^{n}}{n !(n+1) !},
\end{aligned}
$$




$$
\begin{aligned}
& \phi(a, c, x) \underset{\operatorname{Re} x \rightarrow+\infty}{\longrightarrow} \frac{\Gamma(c)}{\Gamma(a)} \mathrm{e}^{x} x^{a-c}(1+\theta(1 /|x|)), \\
& \phi(a, c, x) \underset{\operatorname{Re} x \rightarrow-\infty}{\longrightarrow} \frac{\Gamma(c)}{\Gamma(c-a)}(-x)^{-a}(1+\theta(1 /|x|)),
\end{aligned}
$$

$\phi_{1}(a, b, c, x, y)$ a degenerate hypergeometric series of two variables

$$
\phi_{1}(a, b, c, y)=\sum_{m, n=0}^{\infty} \frac{(a)_{m+n}(b)_{n}}{(c)_{m+n}} x^{m} y^{n}
$$

$B(x, y)$ the Euler function.

We make use of some definite integrals, namely [22]

$$
\begin{aligned}
& \int_{0}^{1} x^{\nu-1}(1-x)^{\mu-1} \mathrm{~d} x=B(\nu, \mu), \quad \int_{0}^{\infty} \mathrm{e}^{-v} v^{x-1} \mathrm{~d} v=\Gamma(x), \\
& \int_{0}^{1} x^{\nu-1}(1 \downarrow x)^{\mu-1} \mathrm{e}^{\beta x} \mathrm{~d} x=B(\nu, \mu) \phi(\nu, \mu+\nu ; \beta), \\
& \int_{0}^{1} x^{\nu-1}(1-x)^{\mu-1}(1-\gamma x)^{-\rho} \mathrm{e}^{\beta x} \mathrm{~d} x=B(\nu, \mu) \phi_{1}(\nu, \rho, \mu+\nu ; \gamma, \beta) .
\end{aligned}
$$

(a) Residues of the poles of $\left(P_{s} C_{t}\right)$. The $\left(P_{s} C_{t}\right)$ term has poles for $s$ such that $\alpha(s)=n, n=1,2, \ldots$ with residues

$$
\begin{aligned}
& R_{n}=\frac{(-)^{n-1}}{(n-1) !} \int_{0}^{\infty} \mathrm{e}^{-\lambda} \mathrm{d} \lambda \int_{0}^{b} \mathrm{~d} \mu \sqrt{\mu(b-\mu)} \frac{\Gamma\left(-\left(\alpha_{0}-1\right)(1+\lambda / q)-\mu t\right)}{\Gamma\left(-\left(\alpha_{0}-1\right)(1+\lambda / q)-\mu t-n\right)} \\
& =\frac{(-)^{n-1}}{(n-1) !} \int_{0}^{\infty} \mathrm{e}^{-\lambda} \mathrm{d} \lambda b^{2} \int_{0}^{1} \mathrm{~d} \mu \sqrt{\mu(1-\mu)} \sum_{m=0}^{n} D_{m}^{n}\left(-\left(\alpha_{0}-1\right)(1+\lambda / q)-\mu b t\right)^{m} \\
& =\frac{(-)^{n-1}}{(n-1) !} b^{2} \sum_{m=0}^{n} D_{m}^{n} \int_{0}^{\infty} \mathrm{e}^{-\lambda} \mathrm{d} \lambda \int_{0}^{1} \mathrm{~d} \mu \sqrt{\mu(1-\mu)} \sum_{k=0}^{m} C_{k}^{m}\left(1-\alpha_{0}\right)^{m-k}(1+\lambda / q)^{m-k}(-b \mu t)^{k} \\
& =\frac{(-)^{n-1}}{(n-1) !} b^{2} \sum_{m=0}^{n} D_{m}^{n} \sum_{k=0}^{m} C_{k}^{m}(-b t)^{m-k}\left(1-\alpha_{0}\right)^{k} B\left(\frac{3}{2}+m-k, \frac{3}{2}\right) \sum_{j=0}^{k} C_{j}^{k} \frac{j !}{q^{j}} .
\end{aligned}
$$


On the leading trajectory, the residues may be obtained from the Veneziano ones (where $b$ would be the slope) by multiplying by a factor $b^{2} B\left(\frac{3}{2}+n, \frac{3}{2}\right)$.

(b) Cut discontinuities. In order to study the cut discontinuities, one can make an expansion of the Euler function

$$
\begin{aligned}
\left(P_{t} C_{s}\right) & =-\sum_{n=1}^{\infty} \frac{(\alpha(t))_{n}}{(n-1) !} \int_{0}^{\infty} \mathrm{e}^{-\lambda} \mathrm{d} \lambda \int_{0}^{b} \mathrm{~d} \mu \sqrt{\mu(b-\mu)} \frac{1}{-\left(\alpha_{0}-1\right)(1+\lambda / q)-\mu s+n-1} \\
& =-\frac{q}{1-\alpha_{0}} \sum_{n=1}^{\infty} \frac{(\alpha(t))_{n}}{(n-1) !} \int_{n-\alpha_{0}}^{\infty} \frac{\mathrm{d} s^{\prime}}{s^{\prime}-s} \int_{n-\alpha_{0}}^{b} \mathrm{~d} \mu \sqrt{\mu(b-\mu)} \mathrm{e}^{-\frac{q}{1-\alpha_{0}}\left(\mu s^{\prime}-n+\alpha_{0}\right)} \\
& =-\frac{q}{1-\alpha_{\mathrm{o}}} \sum_{n=1}^{\infty} \frac{(\alpha(t))_{n}}{(n-1) !} \int_{n-\alpha_{0}}^{\infty} \frac{\mathrm{d} s^{\prime}}{s^{\prime}-s} \frac{2}{3} \frac{\left(s^{\prime} b-n+\alpha_{0}\right)^{\frac{3}{2}}\left(n-\alpha_{\mathrm{o}}\right)^{\frac{1}{2}}}{s^{\prime 2}} \\
& \times \phi_{1}\left(1,-\frac{1}{2}, \frac{5}{2}, \frac{s^{\prime} b-n+\alpha_{0}}{n-\alpha_{0}},-\frac{q}{1-\alpha_{0}}\left(s^{\prime} b-n+\alpha_{0}\right)\right) .
\end{aligned}
$$

So we obtain for the discontinuity of $\left(P_{t} C_{S}\right)$ :

$$
\begin{aligned}
& \Delta_{s}\left(P_{t} C_{s}\right)=-\frac{2}{3} \pi \frac{q}{1-\alpha_{0}} \sum_{n=1}^{\infty} \frac{(\alpha(t))_{n}}{(n-1) !} \frac{\left(s^{\prime} b-n+\alpha_{\mathrm{o}}\right)^{\frac{3}{2}}\left(n-\alpha_{0}\right)^{\frac{1}{2}}}{s^{\prime 2}} \\
& \quad \times \phi_{1}\left(1,-\frac{1}{2}, \frac{5}{2}, \frac{s^{\prime} b-n+\alpha_{0}}{n-\alpha_{0}},-\frac{q}{1-\alpha_{0}}\left(s^{\prime} b-n+\alpha_{0}\right)\right) \theta\left(s^{\prime} b-n+\alpha_{0}\right) .
\end{aligned}
$$

This formula shows the behavior $\left(s-s_{n}\right)^{\frac{3}{2}}$ at the branch points $s_{n}=\left(n-\alpha_{0}\right) / b$, $n=1,2, \ldots$ Similarly, for the $\left(C_{s} C_{t}\right)$ term

$$
\begin{aligned}
& \Delta_{s}\left(C_{s} C_{t}\right)=\pi \frac{q}{1-\alpha_{0}} \sum_{n=1}^{\infty} \frac{(-)^{n}}{(n-1) !} \sum_{m=0}^{n} D_{m}^{n} \sum_{k=0}^{m} C_{k}^{m}\left(\frac{s^{\prime}-t}{s^{\prime}}\right)^{m}\left(n-\alpha_{0}+\frac{n s^{\prime}}{s^{\prime}-t}\right)^{m-k} \\
& \times \frac{\left(s^{\prime} b-n+\alpha_{0}\right)^{\frac{3}{2}+k}\left(n-\alpha_{0}\right)^{\frac{1}{2}}}{s^{\prime 2}} B\left(k+1, \frac{3}{2}\right) \\
& \times \phi_{1}\left(k+1,-\frac{1}{2}, k+\frac{5}{2}, \frac{b s^{\prime}-n+\alpha_{0}}{n-\alpha_{0}},-\frac{q}{1-\alpha_{0}}\left(b s^{\prime}-n+\alpha_{0}\right)\right) \theta\left(s^{\prime} b-n+\alpha_{0}\right) .
\end{aligned}
$$


(c) Asymptotic behaviour $s \rightarrow \pm \infty$, $t$ fixed. When $s \rightarrow+\infty$, the complex contour $\mu$-integration allows us to use the asymptotic form of the $\Gamma$-functions in $\left(P_{t} C_{s}\right)$ and $\left(C_{s} C_{t}\right)$, since the integrand vanishes at the end points. With the weight $\mathrm{e}^{-\lambda^{t}}$, we can show that it is possible to use this asymptotic form for any value of $\lambda \cdot \operatorname{In}\left(P_{s} C_{t}\right)$, the use of the asymptotic form is allowed by the imaginary part of the trajectory

$$
\begin{aligned}
\left(P_{t} C_{s}\right) & \sim \Gamma(1-\alpha(t)) \int_{0}^{\infty} \mathrm{e}^{-\lambda} \mathrm{d} \lambda \int_{0}^{b} \mathrm{~d} \mu \sqrt{\mu(b-\mu)}(-\mu s)^{\alpha(t)} \\
& =b^{2} \Gamma(1-\alpha(t))(-b s)^{\alpha(t)} \int_{0}^{1} \mathrm{~d} \mu \mu^{\frac{1}{2}+\alpha(t)}(1-\mu)^{\frac{1}{2}} \\
& =b^{2} \Gamma(1-\alpha(t)) B\left(\frac{3}{2}+\alpha(t), \frac{3}{2}\right)(-b s)^{\alpha(t)}
\end{aligned}
$$

This form is valid only for $\alpha(t)>-\frac{3}{2}$. For $\alpha(t)<-\frac{3}{2}$, we have to put the parameter $a>0$ (see eq. (5)) and take the suitable limit for $a \rightarrow 0$. But in all cases, the $s$ dependence is given by $(-b s)^{\alpha(t)}$ which shows how the scale parameter is related to $b$, that is the spacing of subsequent thresholds.

(ii) $\left.\quad\left(P_{s} C_{t}\right) \sim \int_{0}^{\infty} \mathrm{e}^{-\lambda} \mathrm{d} \lambda \int_{0}^{b} \mathrm{~d} \mu \sqrt{\mu(b-\mu)(}-\alpha(s)\right)^{1+\left(\alpha_{0}-1\right)(1+\lambda / q)+\mu t} \Gamma\left(-\left(\alpha_{0}-1\right)(1+\lambda / q)-\mu t\right)$

$$
\begin{aligned}
& =b^{2} \int_{0}^{\infty} \mathrm{e}^{-\lambda} \mathrm{d} \lambda \int_{0}^{1} \mathrm{~d} \mu \sqrt{\mu(1-\mu)} \int_{0}^{\infty} \mathrm{e}^{-v} \mathrm{~d} v\left(\frac{v}{-\alpha(s)}\right)^{-\left(\alpha_{0}-1\right)(1+\lambda / q)-\mu b t-1} \\
& =b^{2} \int_{0}^{\infty} \mathrm{e}^{-v} \mathrm{~d} v\left(\frac{v}{-\alpha(s)}\right)^{-\alpha_{0}} \frac{1}{1+\frac{\alpha_{0}-1}{q} \log \left(\frac{v}{-\alpha(s)}\right)^{\frac{1}{8} \pi \phi}\left(\frac{3}{2}, 3 ; \cdots b t \log \left(\frac{v}{-\alpha(s)}\right)\right) .}
\end{aligned}
$$

For the values of $s$ of our interest, $\mu \approx \frac{1}{2} b$ gives the most important contribution to the integral and so we have $(-(s))^{\alpha_{0}}+\frac{1}{2} b^{2} t f(t)$ behaviour. For asymptotic values of $s$, the most important contribution comes from the end points of the $\mu$-integration.

Using (A.13), (A.14) or (A.15) according to the value of $t$, we obtain for small $t$, to first order in $(t \log s)^{2}$

$$
\left(P_{s} C_{t}\right) \sim \frac{1}{8} \pi b^{2} \int_{0}^{\infty} \mathrm{e}^{-v} \mathrm{~d} v\left(\frac{v}{-\alpha(s)}\right)^{-\alpha_{0}-\frac{1}{2} b t} \frac{1}{1+\frac{\alpha_{0}-1}{q} \log \left(\frac{v}{-\alpha(s)}\right)},
$$


for fixed $t<0$

$$
\left(P_{s} C_{t}\right) \sim \frac{1}{2} \sqrt{\pi} b^{2} \int_{0}^{\infty} \mathrm{e}^{-v} \mathrm{~d} v\left(\frac{v}{-\alpha(s)}\right)^{-\alpha_{0}} \frac{\left[b t \log \left(-\frac{v}{-\alpha(s)}\right)\right]^{-\frac{3}{2}}}{1+\alpha_{0}-1}
$$

for fixed $t>0$

$$
\left.\left(P_{s} C_{t}\right) \sim \frac{1}{2} \sqrt{\pi} b^{2} \int_{0}^{\infty} \mathrm{e}^{-v} \mathrm{~d} v\left(\frac{v}{-\alpha(s)}\right)^{-\alpha_{0}-b t} \frac{\left[b t \log \left(\frac{v}{-\alpha(s)}\right)\right]^{-\frac{3}{2}}}{1+\frac{\alpha_{0} \cdot \log (-v)}{q} \log (-\alpha(s)}\right)
$$

One sees on these behaviours that, as fixed negative $t$, the flat cut dominates whereas at fixed positive $t$ it is the one with the slope $b$ which takes over. In the very forward direction one has a transition regime with an effective cut with slope $\frac{1}{2} b$.

(iii) Similarly one obtains

$$
\left(C_{s} C_{t}\right) \sim b^{2} \int_{0}^{\infty} \mathrm{e}^{-v} \mathrm{~d} v \int_{0}^{1} \mathrm{~d} \mu \sqrt{\mu(1-\mu)}\left(\frac{v}{-\mu b s}\right)^{-\alpha_{0}-\mu b t}-\frac{1}{1+\frac{\alpha_{0}-1}{q} \log \left(\frac{v}{-\mu b s}\right)}
$$

\section{Possible modification of threshold positions}

In order to modify the branch point position $\left(s_{n}=\left(n \cdots \alpha_{0}\right) / b\right)$, we can modify the value of $b$. In order to get the same high energy scale parameter and slope of the cuts, we have to modify the $\mu$ weight function in such a way that the effective highenergy $b$-value remains the same. This can be obtained with the weight function $\sqrt{\mu(b-\mu)}(1+c \mu)$ where the parameter $c$ is such that the derivative of this new weight function is zero at the same place as the previous one.

\section{Numerical calculation}

For practical computations, we have used a 8 point Gauss-Laguerre quadrature for the $\lambda$-integration, and a 10 point Gauss-Tchebycheff quadrature for the $\mu$-integration in the complex $\mu$-plane.

\section{REFERENCES}

[1] G. Cohen-Tannoudji, F.S. Henyey, G.L. Kane and W. Zakrzewski, Phys. Rev. Letters 26 (1971) 112 . 
|2| I.S. Henyey, G.L. Kane, J. Pumplin and M.H. Ross, Phys. Rev. 182 (1969) 1579.

[3] M.H. Ross, F.S. Henyey and G.L. Kane, Nucl. Phys. B23 (1970) 269.

14| V. Barger and L. Durand, Phys. Rev. 156 (1967) 1525.

[5] M.II. Ross, to be published;

B.J. Hartley and G.L. Kane, to be published.

[6| R. Hong Tuan, J.M. Kaplan and G. Sanguinetti, Regge cuts and high energy $\pi \mathrm{N}$ and $\mathrm{KN}$ scattering, Preprint LPTHE 71/14, Orsay.

[7| C. Schmid, Phys, Rev. Letters 20 (1968) 689.

[8] R. Dolen, D. Horn and C. Schmid, Phys. Rev. 166 (1968) 1768.

[9| li. Halzen and C. Michael, Phys. Letters 36B (1971) 367.

10| J.H. Schwarz, private communication;

D.I. Gross, A. Neveu, J. Scherk and J.H. Schwarz, Phys. Rev. D2 (1970) 697.

11] M.O. Taha, Phys. Rev. D3 (1971) 498.

12] see ref. [1];

A.I. Bugrij, L.L. Jenkowsky and N.A. Kobylinsky, Preprints ITP71-28E and 73E, Dual models with Mandelstam analyticity;

M.G. Schmidt, Preprint (Universitat Heidelberg), A dual amplitude with Mandelstam analyticity and Veneziano limit;

P. Olesen, Preprint THI 1376 - CERN, Inclusive reaction in dual models with second sheet resonances;

R. Ramachandran and M.O. Taha, Preprint IC-71-30, Trieste, Dual amplitude with arbitrary trajectories;

L. Gonzales Mestres, Preprint LPTIIF, 71, 126, Orsay, Multipole-free dual amplitudes wit! Mandelstam analyticity;

R. Garkell and A.P. Contogouris, Preprint Mc Gill University, Montreal, Veneziano-like representation with finite width resonances.

131 Proc, of the Conf. on $\pi \pi$ and $\mathrm{K} \pi$ interactions, Argonne, 1969;

J.L. Basdevant and J. Reignier, Lectures at Herceg Novi School, 1970.

14| C. Lovelace, Phys. Letters 28 B (1968) 264.

15| P.G.O. Freund, Phys. Rev. Letters 20 (1968) 235:

H. Harari, Phys. Rev. Letters 20 (1968) 1395.

16] A. Martin. Phys. Letters 29 B (1969) 431.

17| R. Odorico, Crucial tests of the Veneziano model from dip behaviour at low energy, Preprint TH 1303 CERN.

$18 \mid$ Y. Gell, D. Horn, M. Jacob and J. Weyers, Nucl. Phys. B33 (1971) 379.

$19 \mid$ see ref. $[14]$;

C. Altarelli and II. Rubinstein, Phys. Rev. 183 (1969) 1469;

C.P. Gopal, R. Migneron and A. Rothery, Phys. Rev. D3 (1971) 2262;

S. Pokorski, R.O. Raitio and G.M. Thomas, Duality and final state interaction in the description of $\bar{p} n \rightarrow \pi^{+} \pi^{-} \pi^{--}$at rest, Preprint 8-71, Helsinki.

20) M.A. Virasoro, Phys. Rev. 177 (1969) 2309.

21] Higher transcendental functions, vol. 1, (Bateman Manuscript Project).

22] I.S. Gradshteyn and I.M. Ryzkhik, Tables of integrals, series and products, (Academic Press, New York, 1965). 